\title{
A phase I trial of low-dose inhaled carbon monoxide in sepsis-induced ARDS
}

Role of funding source: This study was funded by the NIH. The funding organization had no role in study design, data collection, data analysis, data interpretation, or preparation of the manuscript.

Conflict of interest: LEF has received clinical trial support for an unrelated study from Asahi Kasei Pharma America (AKPA). DRH is a consultant for Philips Respironics and Ventec Life Support; receives publishing royalties from Jones and Bartlett, McGraw-Hill, and UpToDate; and is managing editor of Respiratory Care (Daedalus Enterprises) and an inter-professional member of the Pulmonary Disease Board of the American Board of Internal Medicine. BDK has received grants from Karius Inc. Savara Pharmaceuticals, and Defense Advanced Research Projects Agency, as well as personal fees from La Jolla Pharmaceutical Company. RSH is an employee at Vertex Pharmaceuticals as of December 2017. JDD is a consultant for Teleflex Medical. PFN has received personal fees from Third Pole Inc. CNS has received personal fees from Corbus Pharmaceuticals, Inflammation Research Foundation, and FASEB Journal, as well as grants from Solutex. CNS is an inventor on patents related to resolvins and other pro-resolving mediators (both composition of matter and use of) that are licensed by Partners-Brigham and Women's Hospital (Partners-BWH) for clinical development. BTT has served as a consultant on ARDS clinical trial design for Bayer, Boehringer Ingelheim, and ClaxoSmithKline. AMKC is a cofounder of and SAB member for Proterris Inc. and served as a consultant for Teva Pharmaceuticals. AMKC has a use patent on $\mathrm{CO}$, which belongs to University of Pittsburgh, Johns Hopkins University, Yale University, and Proterris Inc.

License: Copyright 2018, American Society for Clinical Investigation.

Submitted: August 6, 2018 Accepted: October 29, 2018 Published: December 6, 2018

Reference information: JCI Insight. 2018;3(23):e124039. https://doi.org/10.1172/jci. insight.124039.
Laura E. Fredenburgh, ${ }^{1}$ Mark A. Perrella, ${ }^{1,2}$ Diana Barragan-Bradford, ${ }^{1}$ Dean R. Hess, ${ }^{3,4}$ Elizabeth Peters, ${ }^{5}$ Karen E. Welty-Wolf, ${ }^{6}$ Bryan D. Kraft, ${ }^{6}$ R. Scott Harris, ${ }^{7}$ Rie Maurer, ${ }^{8}$ Kiichi Nakahira, ${ }^{5}$ Clara Oromendia, ${ }^{9}$ John D. Davies, ${ }^{10}$ Angelica Higuera, ${ }^{1}$ Kristen T. Schiffer, ${ }^{5}$ Joshua A. Englert, ${ }^{1}$ Paul B. Dieffenbach, ${ }^{1}$ David A. Berlin, ${ }^{5}$ Susan Lagambina, ${ }^{11}$ Mark Bouthot, ${ }^{11}$ Andrew I. Sullivan, ${ }^{11}$ Paul F. Nuccio, ${ }^{11}$ Mamary T. Kone, ${ }^{7}$ Mona J. Malik, ${ }^{6}$ Maria Angelica Pabon Porras, ${ }^{5}$ Eli Finkelsztein, ${ }^{5}$ Tilo Winkler, ${ }^{3}$ Shelley Hurwitz, ${ }^{8}$ Charles N. Serhan, ${ }^{12}$ Claude A. Piantadosi, ${ }^{6}$ Rebecca M. Baron, ${ }^{1}$ B. Taylor Thompson, ${ }^{7}$ and Augustine M.K. Choi ${ }^{5}$

'Division of Pulmonary and Critical Care Medicine, Department of Medicine, Brigham and Women's Hospital, Boston, Massachusetts, USA. Department of Pediatric Newborn Medicine, Brigham and Women's Hospital, Boston, Massachusetts, USA. ${ }^{3}$ Department of Anesthesia, Critical Care, and Pain Medicine, Massachusetts General Hospital, Boston, Massachusetts, USA. ${ }^{4}$ Department of Respiratory Care, Massachusetts Ceneral Hospital, Boston, Massachusetts, USA. ${ }^{5}$ Division of Pulmonary and Critical Care Medicine, Department of Medicine, Weill Cornell Medicine, New York, New York, USA. ${ }^{6}$ Division of Pulmonary, Allergy, and Critical Care Medicine, Department of Medicine, Duke University Medical Center, Durham, North Carolina, USA. 'Division of Pulmonary and Critical Care Medicine, Department of Medicine, Massachusetts General Hospital, Boston, Massachusetts, USA. ${ }^{8}$ Department of Medicine, Brigham and Women's Hospital, Boston, Massachusetts, USA. 'Department of Healthcare Policy and Research, Division of Biostatistics and Epidemiology, Weill Cornell Medicine, New York, New York, USA. ${ }^{10}$ Department of Respiratory Care, Duke University Medical Center, Durham, North Carolina, USA. "Department of Respiratory Care, Brigham and Women's Hospital, Boston, Massachusetts, USA. ${ }^{12}$ Center for Experimental Therapeutics and Reperfusion Injury, Department of Anesthesiology, Perioperative and Pain Medicine, Brigham and Women's Hospital, Boston, Massachusetts, USA.

BACKCROUND. Acute respiratory distress syndrome (ARDS) is a prevalent disease with significant mortality for which no effective pharmacologic therapy exists. Low-dose inhaled carbon monoxide (iCO) confers cytoprotection in preclinical models of sepsis and ARDS.

METHODS. We conducted a phase I dose escalation trial to assess feasibility and safety of low-dose iCO administration in patients with sepsis-induced ARDS. Twelve participants were randomized to iCO or placebo air 2:1 in two cohorts. Four subjects each were administered iCO (100 ppm in cohort 1 or $\mathbf{2 0 0 ~ p p m ~ i n ~ c o h o r t ~ 2 ) ~ o r ~ p l a c e b o ~ f o r ~} \mathbf{9 0}$ minutes for up to $\mathbf{5}$ consecutive days. Primary outcomes included the incidence of carboxyhemoglobin ( $\mathrm{COHb}$ ) level $\geq 10 \%$, prespecified administrationassociated adverse events (AEs), and severe adverse events (SAEs). Secondary endpoints included the accuracy of the Coburn-Forster-Kane (CFK) equation to predict COHb levels, biomarker levels, and clinical outcomes.

RESULTS. No participants exceeded a COHb level of $10 \%$, and there were no administrationassociated AEs or study-related SAEs. CO-treated participants had a significant increase in COHb (3.48\% $\pm 0.7 \%$ [cohort 1]; $4.9 \% \pm 0.28 \%$ [cohort 2]) compared with placebo-treated subjects $(1.97 \%$ $\pm 0.39 \%)$. The CFK equation was highly accurate at predicting $\mathrm{COHb}$ levels, particularly in cohort $2\left(R^{2}=0.9205 ; P<0.0001\right)$. Circulating mitochondrial DNA levels were reduced in iCO-treated participants compared with placebo-treated subjects.

CONCLUSION. Precise administration of low-dose iCO is feasible, well-tolerated, and appears to be safe in patients with sepsis-induced ARDS. Excellent agreement between predicted and observed $\mathrm{COHb}$ should ensure that $\mathrm{COHb}$ levels remain in the target range during future efficacy trials.

TRIAL REGISTRATION. ClinicalTrials.gov NCT02425579.

FUNDING. NIH grants P01HL108801, KL2TR002385, K08HL130557, and K08GM102695. 


\section{Introduction}

Acute respiratory distress syndrome (ARDS) is a highly prevalent disease characterized by severe acute lung injury (ALI) and hypoxemic respiratory failure, with overall mortality rates of $40 \%(1)$. Sepsis is a major risk factor for ARDS and multiple organ dysfunction with unacceptably high morbidity and mortality $(2,3)$. Despite advances in critical care management and lung-protective ventilation strategies (4-6), treatment for sepsis-induced ARDS remains supportive, and there is an urgent need for new therapies targeting novel pathways.

Low-dose inhaled carbon monoxide (iCO) is a promising new therapy for sepsis-induced ARDS supported by compelling data from preclinical models (7-14). CO is a gas produced endogenously by the heme oxygenase (HO) enzymes that exerts numerous biologic effects, including protection against cell death, suppression of inflammation, attenuation of edema, and enhancement of phagocytosis (7-14), all of which are critical in the resolution of sepsis-induced ARDS. We have also recently found several new mechanisms by which CO exerts protection, including induction of autophagy $(11,15)$, inhibition of mitochondrial dysfunction and inflammasome activation (16), activation of mitochondrial biogenesis $(9,17)$, and acceleration of inflammation resolution via biosynthesis of specialized pro-resolving mediators (SPMs) (9, 18, 19).

Low-dose iCO therapy has been shown to be safe and well tolerated in healthy volunteers $(17,20-26)$ and patients with chronic lung disease $(27,28)$. We have recently developed and tested a novel ventilator-compatible $\mathrm{CO}$ delivery system and a $\mathrm{CO}$ dosing strategy in a baboon model of $S$. pneumoniae pneumonia (9). We demonstrated that low-dose iCO (100-300 ppm) can be safely administered to mechanically ventilated baboons with ALI, and that carboxyhemoglobin $(\mathrm{COHb})$ levels can be accurately predicted using the Coburn-Forster-Kane $(\mathrm{CFK})$ equation $\left(R^{2}=0.9864\right)(9)$. The CFK equation, a well-validated model of $\mathrm{CO}$ uptake, accurately predicts the rise in $\mathrm{COHb}$ following $\mathrm{CO}$ exposure in humans with normal lung function $(22,29,30)$, but had never been used in mechanically ventilated patients with impaired gas exchange. We also found that CO attenuates pneumonia-induced ALI in baboons by activating lung mitochondrial biogenesis (9), reducing proinflammatory urinary cysteinyl leukotrienes (31), and partially restoring levels of circulating SPMs (19). Based on these studies, we aimed to assess the feasibility and safety of low-dose iCO administration in patients with sepsis-induced ARDS. We also evaluated the accuracy of the CFK equation in predicting $\mathrm{COHb}$ levels in patients with sepsis-induced ARDS.

\section{Results}

Between April 28, 2015 and November 14, 2016, 145 patients were assessed for study eligibility. Of those screened, 133 patients were ineligible, and 12 eligible participants were enrolled and randomized between July 10, 2015, and November 14, 2016 (Figure 1). In cohort 1, four subjects were randomized to and received iCO (100 ppm), and two subjects were assigned to and received placebo air. In cohort 2, four subjects were assigned to and received iCO (200 ppm), and two subjects were randomized to and received air. All participants received at least one dose of the study drug and were included in the primary endpoint analysis.

Baseline demographics are shown in Table 1 . The mean age $( \pm S D)$ of participants in cohort 1 was 57 \pm 19 years, while the mean age of participants in cohort 2 was $49 \pm 9$ years. Baseline clinical characteristics based on treatment assignment (iCO 100, iCO 200, combined placebo groups) are shown in Table 2, and baseline characteristics for individual participants are shown in Table 3. Most patients had pneumonia as the primary source of infection, and the majority of subjects had confirmed infection based on microbiologic data (Supplemental Table 1; supplemental material available online with this article; https://doi.org/10.1172/jci.insight.124039DS1). Baseline lactate was significantly higher in patients randomized to $100 \mathrm{ppm}$ iCO compared with $200 \mathrm{ppm}$ iCO- and placebo-treated patients $(P=0.0092)$; however, this was likely a stochastic artifact of the small sample size. There were no other significant differences in baseline characteristics among the treatment groups.

All subjects tolerated the study drug treatment and study procedures well, and no participants required stopping the study drug earlier than 90 minutes. In participants treated with $100 \mathrm{ppm}$ iCO, the median partial pressure of arterial oxygen $\left(\mathrm{PaO}_{2}\right)$ was $98.5 \mathrm{mmHg}(\mathrm{IQR} 73.3,111.8)$ and $90.5 \mathrm{mmHg}(\mathrm{IQR} 82.8$, 114.8) before treatment and 90 minutes after treatment, respectively. In participants treated with $200 \mathrm{ppm}$ iCO, the median $\mathrm{PaO}_{2}$ was $104 \mathrm{mmHg}$ (IQR 92, 123) and $108 \mathrm{mmHg}$ (IQR 79.5, 118.5) before treatment and after treatment, respectively. In placebo-treated subjects, the median $\mathrm{PaO}_{2}$ was $86 \mathrm{mmHg}$ (IQR 83, 105) and $86 \mathrm{mmHg}(\mathrm{IQR} \mathrm{76,93)} \mathrm{before} \mathrm{treatment} \mathrm{and} \mathrm{after} \mathrm{treatment,} \mathrm{respectively.} \mathrm{The} \mathrm{partial} \mathrm{pressure} \mathrm{of}$ arterial carbon dioxide $\left(\mathrm{PaCO}_{2}\right)$, arterial oxygen saturation $\left(\mathrm{SaO}_{2}\right)$, and arterial $\mathrm{pH}$ were similar among the 


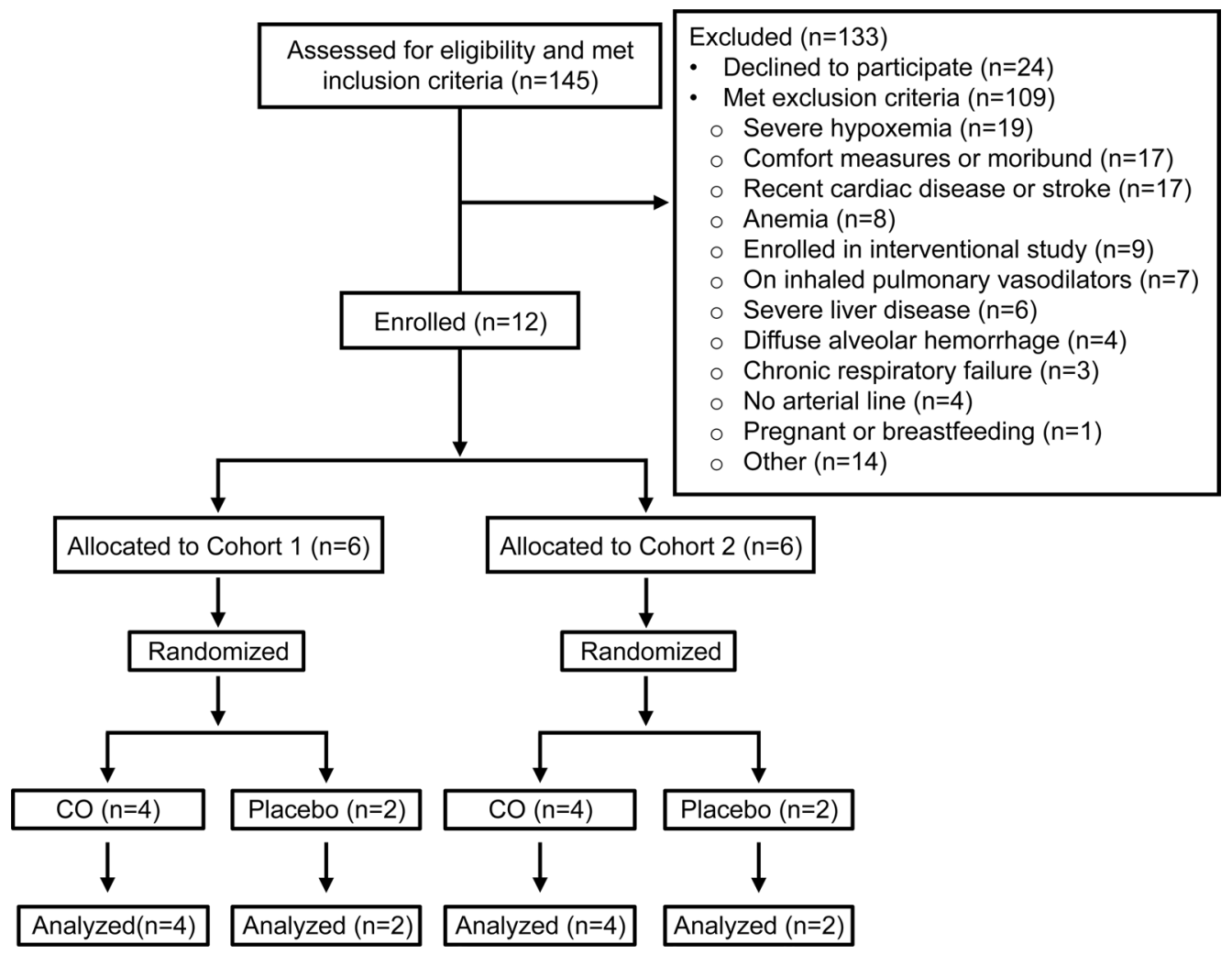

Figure 1. CONSORT diagram. CONSORT subject flow diagram shows the number of subjects screened, enrolled, randomized, and included in the primary analysis. One hundred forty-five patients were screened, and 12 participants were enrolled. In cohort 1, 4 subjects were randomized to $100 \mathrm{ppm}$ iCO and 2 subjects were randomized to placebo air. In cohort 2, 4 subjects were randomized to $200 \mathrm{ppm}$ iCO and 2 subjects were randomized to placebo air. The primary safety endpoint analysis included all subjects treated with at least one dose of iCO or placebo.

treatment groups and did not significantly change following treatment. Arterial blood gas parameters for individual participants before and after treatment are shown in Figure 2 and Supplemental Table 2. Arterial blood gas parameters for individual participants before and after treatment on each day of treatment are shown in Supplemental Figures 1 and 2.

Subjects received the study drug for a minimum of 2 days and a maximum of 4 days in both cohorts (median treatment 2.5 days [IQR 2, 4] in cohort 1; 3.5 days [IQR 2, 4] in cohort 2). Doses were planned to be given at 24-hour intervals and were typically given within 1 hour clock time of the preceding dosage. No subjects in either cohort met prespecified safety criteria for permanent discontinuation of the study drug. Reasons for the study drug holds are shown in Supplemental Table 3. The most common reason that subjects did not receive treatment was discontinuation of mechanical ventilation, which was a prespecified study treatment endpoint. Additional reasons included low hemoglobin ( $\mathrm{Hgb})$, loss of required arterial line (to monitor arterial $\mathrm{COHb}$ levels), and severe hypoxemia, all of which were prespecified in the protocol of this phase I safety trial. One CO-treated participant (subject 10 in cohort 2) had a baseline COHb of $3.27 \%$ on study day 5 . The elevated baseline $\mathrm{COHb}$ level on day 5 was thought to most likely reflect increased endogenous $\mathrm{CO}$ production secondary to upregulated HO-1 expression (32), rather than delayed CO clearance, as the estimated CO half-life (33) in this patient was approximately 93 minutes.

Baseline $\mathrm{COHb}$ levels were not different across treatment groups. The mean $( \pm \mathrm{SD}) \mathrm{COHb}$ at 90 minutes in CO-treated subjects was $3.48 \% \pm 0.7 \%$ in cohort 1 and $4.9 \% \pm 0.28 \%$ in cohort 2 on day 1 (Figure 3 and Supplemental Tables 4 and 5). COHb levels were significantly different between groups over time on days 1-3 by 2-way ANOVA. Adjusted $P$ values from Tukey's post hoc tests are shown in Supplemental Table 6.

The maximum $\mathrm{COHb}$ level in cohort 1 was $4.4 \%$ and $6.87 \%$ in cohort 2 . The maximum change in $\mathrm{COHb}$ from baseline to 90 minutes $(\triangle 90 \mathrm{~min} \mathrm{COHb}$ ) was $2.5 \%$ in cohort 1 and $4 \%$ in cohort 2 . COHb levels for individual participants on each day are shown in Supplemental Figures 3 and 4. Repeated-measures ANOVA found a significant treatment effect in $\Delta 90 \mathrm{~min} \mathrm{COHb}$ in both cohort $1(P=0.0098)$ and cohort $2(P=$ 0.0018). However, there was no significant difference in $\Delta 90 \mathrm{~min} \mathrm{COHb}$ by day and no interaction effect between day and treatment in either cohort.

No subjects exceeded a $\mathrm{COHb}$ level of $7 \%$, which is well below our prespecified safety threshold of $10 \%$, and no administration-associated AEs were observed. A total of 17 nonserious AEs and SAEs from 9 subjects were reported. The majority of nonserious AEs (67\% [6 of 9]) were rated as moderate (National 
Table 1. Baseline demographics of participants by cohort

\begin{tabular}{lccc}
\hline & Cohort 1 $(\boldsymbol{n}=\mathbf{6})$ & Cohort 2 (n= 6) & P value \\
Female sex, no. (\%) & $3(50 \%)$ & $3(50 \%)$ & 1.0000 \\
Age (yr) & $57 \pm 19$ & $49 \pm 9$ & 0.1039 \\
Race, no. (\%) & & $6(100 \%)$ & \\
$\quad$ White & $4(66.7 \%)$ & 0 & \\
Black & $2(33.3 \%)$ & $5(83.3 \%)$ & \\
Ethnicity, no. (\%) & $6(100 \%)$ & $1(16.7 \%)$ & \\
$\quad$ Not Hispanic or Latino & 0 & & \\
Hispanic or Latino & & \\
Plus-minus values are mean \pm SD. & &
\end{tabular}

Cancer Institute Common Terminology Criteria for Adverse Events [CTCAE] grade 2) or lower. Three SAEs occurred in the CO treatment arm, and 5 SAEs occurred in the placebo arm, none of which were judged to be study related. One participant died in cohort 1 on study day 8 after the legally authorized representative (LAR) made the decision to transition to comfort measures. The death was reported in an expedited fashion to the Data Coordinating Center (DCC; members from BWH and Massachusetts General Hospital $[\mathrm{MGH}]$ ) and the Data and Safety Monitoring Board (DSMB), which reviewed the case in detail and concurred with the medical monitor assessment that it was unrelated to study participation. All SAEs were independently reviewed by the Scientific Review Committee (SRC) and DSMB, which agreed with the investigators' assessments that none were related to study drug administration. Details of the SAEs are shown in Table 4. Clinical outcomes, vital status, and study day of discharge for each subject are presented in Table 5 .

Baseline and 20-minute-measured $\mathrm{COHb}$ levels were input into the $\mathrm{CFK}$ equation to predict what $\mathrm{COHb}$ levels would be after 60,75 , and 90 minutes of iCO treatment. We found an excellent correlation between measured and predicted $\mathrm{COHb}$ levels in participants treated with $100 \mathrm{ppm}$ iCO (Spearman's $r=$ 0.8614; $P<0.0001$ ) (Figure 4A) and an even stronger correlation in subjects treated with $200 \mathrm{ppm}$ iCO (Spearman's $r=0.916 ; P<0.0001$ ) (Figure 4B). Modeling the cohort 1 data with linear regression revealed a slope of $0.7778(95 \%$ CI $[0.6082,0.9475])$, a $y$-intercept of $0.6558(95 \%$ CI $[0.05005,1.262])$, and goodness-of-fit $R^{2}=0.7186(P<0.0001)$. Modeling the cohort 2 data with linear regression revealed a slope of 1.112 (95\% CI $[1.003,1.22])$, a $y$-intercept of -0.6632 (95\% CI [-1.174, -0.1522$])$, and goodness-of-fit $R^{2}$ $=0.9204(P<0.0001)$. Bland-Altman plots also demonstrated excellent agreement between measured and CFK equation-predicted $\mathrm{COHb}$ levels (Figure 4, C and D) with a mean difference between measured and predicted $\mathrm{COHb}$ levels of $0.1279 \pm 0.3023$ in cohort 1 and $0.1467 \pm 0.2738$ in cohort 2 .

\section{Table 2. Baseline clinical characteristics of treatment groups}

\begin{tabular}{|c|c|c|c|c|}
\hline & iCO 100 ppm (n = 4) & iCO 200 ppm (n = 4) & Placebo $(n=4)$ & P value \\
\hline $\begin{array}{l}\text { Interval between ARDS } \\
\text { onset and enrollment (h) }\end{array}$ & $22.1 \pm 24.5$ & $55.1 \pm 44.4$ & $55.8 \pm 21.3$ & 0.3475 \\
\hline APACHE II score & $28.8 \pm 9.9$ & $23.5 \pm 12.2$ & $30 \pm 10.8$ & 0.7465 \\
\hline SOFA score & $11.3 \pm 2.2$ & $10.3 \pm 3.5$ & $13.3 \pm 4.3$ & 0.5893 \\
\hline Lactate & $7.15 \pm 9.47$ & $1.03 \pm 0.24$ & $1.13 \pm 0.25$ & 0.0092 \\
\hline Tidal volume (ml/kg/PBW) & $6.53 \pm 0.34$ & $7.57 \pm 1.36$ & $5.6 \pm 0.78$ & 0.0545 \\
\hline Plateau pressure $\left(\mathrm{cm} \mathrm{H}_{2} \mathrm{O}\right)$ & $18.5 \pm 4.2$ & $25.8 \pm 4.6$ & $28.5 \pm 7.8$ & 0.0719 \\
\hline $\operatorname{PEEP}\left(\mathrm{cm} \mathrm{H}_{2} \mathrm{O}\right)$ & $8.3 \pm 2.4$ & $12.5 \pm 3.4$ & $15 \pm 4.8$ & 0.0705 \\
\hline $\mathrm{PaO}_{2} / \mathrm{FiO}_{2}(\mathrm{mmHg})$ & $143 \pm 75$ & $166 \pm 59$ & $110 \pm 38$ & 0.3070 \\
\hline Ol & $11.5 \pm 7$ & $11.2 \pm 5.3$ & $21.9 \pm 10.8$ & 0.2601 \\
\hline LIS & $2.56 \pm 0.31$ & $2.81 \pm 0.47$ & $3.31 \pm 0.43$ & 0.0729 \\
\hline
\end{tabular}

APACHE, acute physiology and chronic health evaluation; LIS, lung injury score; OI, oxygenation index; PBW, predicted body weight; PEEP, positive endexpiratory pressure; SOFA, sequential organ failure assessment. Plus-minus values are mean \pm SD. 
Table 3. Baseline characteristics of individual participants

\begin{tabular}{|c|c|c|c|c|c|c|c|c|c|c|c|}
\hline Subject & Treatment & Site of infection & APACHE II & SOFA & Lactate & $\begin{array}{l}\text { Tidal volume } \\
\text { (ml/kg/PBW) }\end{array}$ & $\begin{array}{c}\text { Plateau pressure } \\
\left(\mathrm{cm} \mathrm{H} \mathrm{H}_{2} \mathrm{O}\right)\end{array}$ & $\begin{array}{c}\text { PEEP } \\
\left(\mathrm{cm} \mathrm{H} \mathrm{H}_{2} \mathrm{O}\right)\end{array}$ & $\begin{array}{l}\mathrm{PaO}_{2} / \mathrm{FiO}_{2} \\
(\mathrm{mmHg})\end{array}$ & 이 & LIS \\
\hline \multicolumn{12}{|l|}{ Cohort 1} \\
\hline Subject 1 & CO & Lung/pleura & 37 & 14 & 21.3 & 6.67 & 24 & 10 & 104 & 13.5 & 3 \\
\hline Subject 2 & Air & Lung/pleura & 42 & 19 & 1.2 & 4.77 & 34 & 12 & 74 & 29.7 & 3.75 \\
\hline Subject 3 & CO & Lung/pleura & 37 & 12 & 2.0 & 6.94 & 14 & 10 & 63 & 20.6 & 2.5 \\
\hline Subject 4 & CO & Lung/pleura & 24 & 10 & 3.6 & 6.26 & 19 & 8 & 235 & 5.5 & 2.25 \\
\hline Subject 6 & Air & GI/biliary tract & 33 & 14 & 1.1 & 5.15 & 27 & 20 & 81 & 31.9 & 3.5 \\
\hline \multicolumn{12}{|l|}{ Cohort 2} \\
\hline Subject 7 & Air & Lung/pleura & 29 & 10 & 0.8 & 6.02 & 18 & 10 & 142 & 9.2 & 2.75 \\
\hline Subject 8 & CO & Lung/pleura & 21 & 9 & 1.2 & 6.51 & 20 & 12 & 100 & 16 & 2.5 \\
\hline Subject 9 & CO & Lung/pleura & 37 & 14 & 1.2 & 8.20 & 31 & 16 & 146 & 14.4 & 3.5 \\
\hline
\end{tabular}

APACHE, acute physiology and chronic health evaluation; LIS, lung injury score; OI, oxygenation index; PBW, predicted body weight; PEEP, positive endexpiratory pressure; SOFA, sequential organ failure assessment.

Analysis of additional secondary outcomes showed that the $\mathrm{PaO}_{2} / \mathrm{FiO}_{2}$ (ratio of the $\mathrm{PaO}_{2}$ to the fraction of inspired oxygen $\left[\mathrm{FiO}_{2}\right]$ ) increased and oxygenation index (OI) decreased over time in all groups, but the difference among groups was not significant (Figure 5, A and B). The mean lung injury score (LIS) declined between baseline and day 7 in iCO-treated and placebo subjects (Figure 5C). Using a mixed-model analysis, there was a highly significant difference in LIS over time $(P<0.0001)$ and a trend toward a difference between the treatment groups $(P=0.1731)$, but no significant difference when the treatment groups were analyzed over time. Lactate levels declined over time in all groups, but the difference among groups over time was not significant (Figure 5D). Mean sequential organ failure assessment (SOFA) scores also decreased between baseline and day 7 in all treatment groups, with the greatest decline in iCO-treated subjects in cohort 1 (11.25 to 2) compared with iCO-treated patients in cohort 2 (10.25 to 5.25) and placebo-treated (13.25 to 7.25) subjects (Figure 5E). Using an ordinal logistic analysis as described previously (34-36), we found a significant difference in SOFA scores over time $(P=0.0145)$ and a trend toward a difference between the treatment groups $(P=0.1173)$, but no significant difference when the treatment groups were analyzed over time.

For exploratory secondary biomarker endpoints, we analyzed the plasma concentrations of mitochondrial DNA (mtDNA), receptor-interacting serine/threonine-protein kinase 3 (RIPK3), and IL-18 in iCO-treated and placebo subjects at baseline prior to treatment on study day 1 and after treatment on days 1-5. These biomarkers were chosen given their association with mortality or disease severity in critical illness (37-39). The median baseline mtDNA plasma level among participants was 5,655 $\pm 10,994$ copies/ $\mu 1$, which is similar to levels previously observed in critically ill patients with sepsis $(4,675 \pm 24,069$ copies/ $\mu$ l) and sepsis/ARDS $(11,662 \pm 25,251$ copies/ $\mu 1)(38)$. Plasma levels of mtDNA were not significantly different among the treatment groups at baseline (Supplemental Figure 5A and Supplemental Table 7). However, on day 2, after two doses of the study drug, iCO-treated patients had significantly reduced mtDNA levels versus their pretreatment baseline levels, when compared with the difference in placebo recipients. Placebo-treated patients had a mean $400 \%$ increase in plasma mtDNA from baseline to day 2, compared with iCO-treated subjects in cohort 1 , who had a mean increase of $11 \%$ in mtDNA from baseline to day $2(P=0.039$ vs. placebo), and iCO-treated subjects in cohort 2 , who had an $88 \%$ decrease in mtDNA ( $P=0.003$ vs. placebo) from baseline to day 2 (Figure 6A and Supplemental Figure 6). A sensitivity analysis using nonparametric Kruskal-Wallis tests showed consistent results, suggesting these differences were not driven by outliers. Similar trends were observed in iCO-treated patients on subsequent days of treatment (Supplemental Figure 7). Of note, subject 10, who was excluded from this analysis, had a $56.4 \%$ decrease in mtDNA after one dose of 200 ppm iCO, which was similar to the decrease observed in the other 200 ppm iCO-treated subjects. 
A $\cdot \Delta \cdot$ Placebo (Cohorts 1 and 2$)$

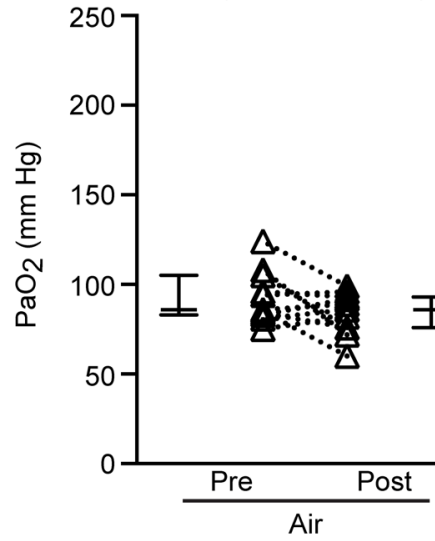

iCO 100 ppm (Cohort 1)

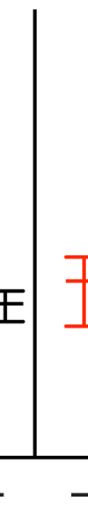

iCO 200 ppm (Cohort 2)

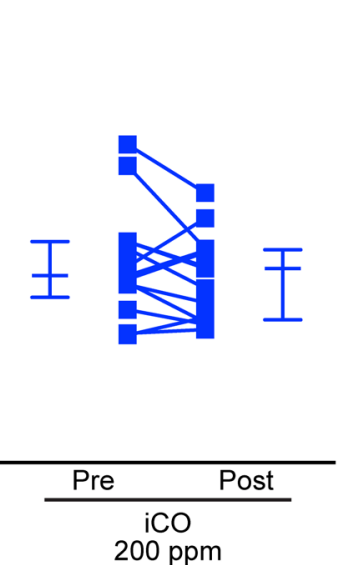

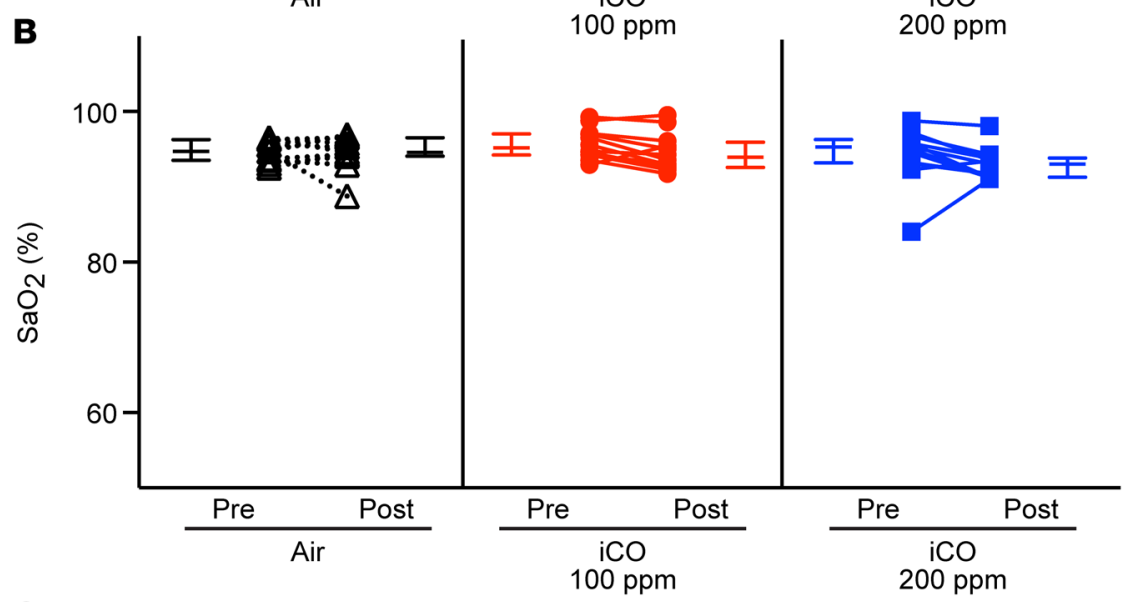
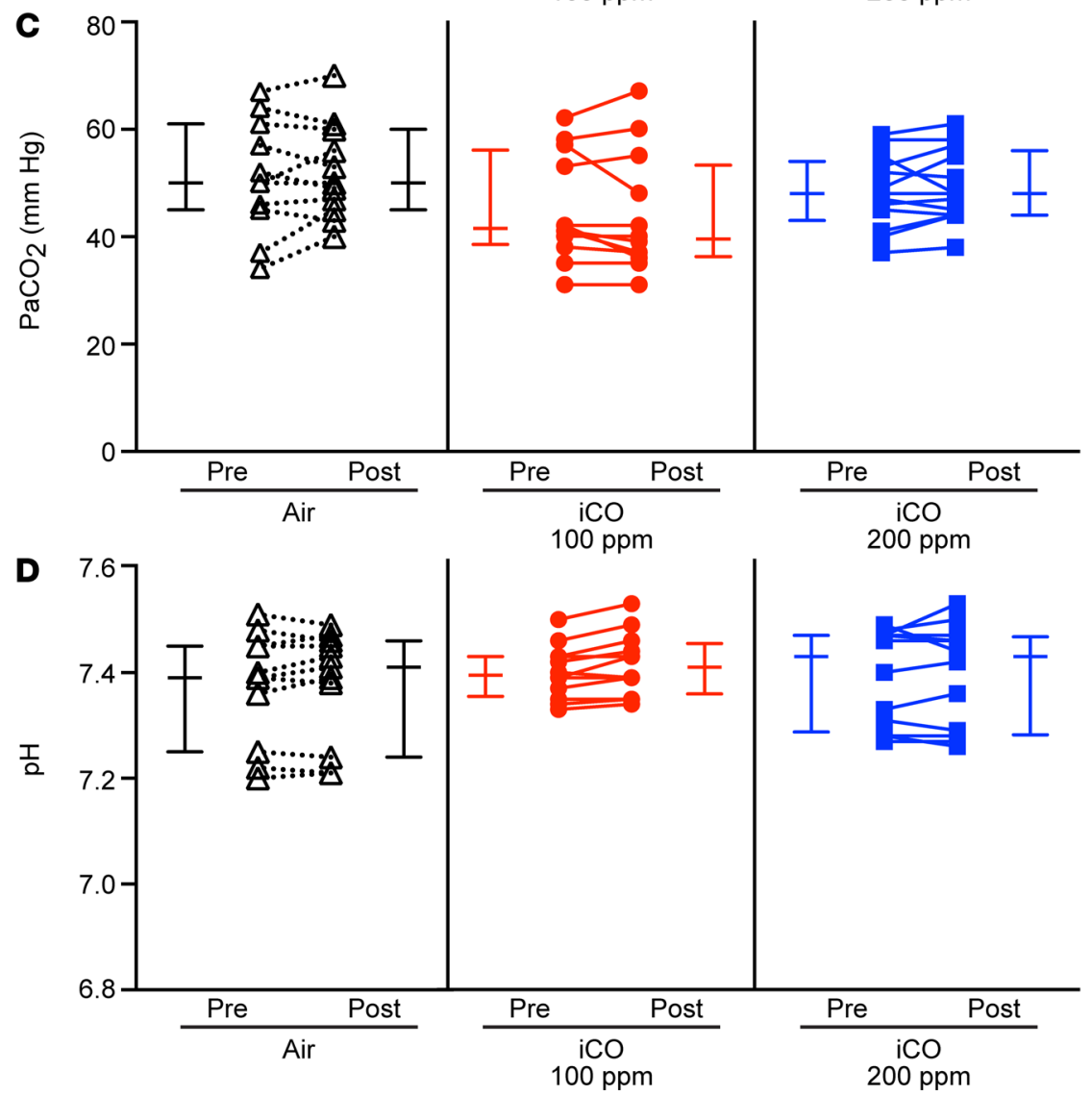

Figure 2. Arterial blood gas parameters before and after treatment with iCO versus placebo air. Arterial blood was drawn from participants before treatment (Pre) and 90 minutes following treatment (Post) with placebo air (day $1, n$ = 4; day $2, n=4$; day $3, n=2$; day $4, n=1$ ), iCO $100 \mathrm{ppm}$ (day $1, n=4$; day $2, n=4$; day $3, n=2$; day $5, n=2$ ), or iCO 200 ppm (day $1, n=2$; day 2, $n=4$; day $3, n=2$; day $4, n=4$; day $5, n=1$ ). Pre- and post-treatment values of $\mathrm{PaO}_{2}(\mathbf{A})$, $\mathrm{SaO}_{2}$ (B), $\mathrm{PaCO}_{2}$ (C), and pH (D) for each participant on each day of treatment. Error bars represent the median and interquartile range. 
A

\section{Day 1}

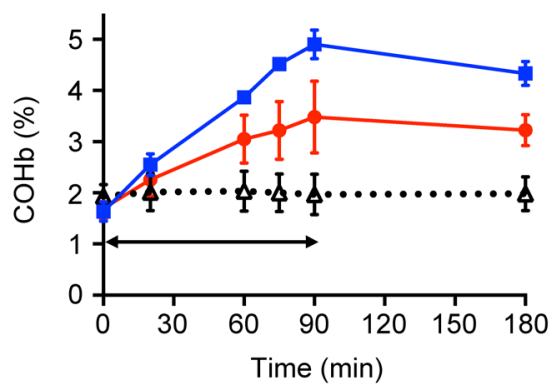

D

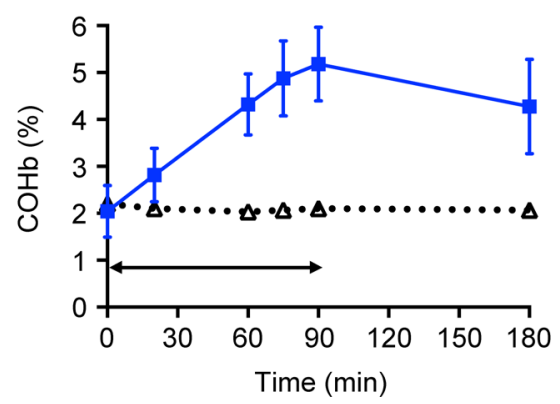

$\mathrm{p}<0.0001$ time $\mathrm{p}=0.002$ treatment $p<0.0001$ interaction
B Day $2 \quad p=0.0044$ treatment

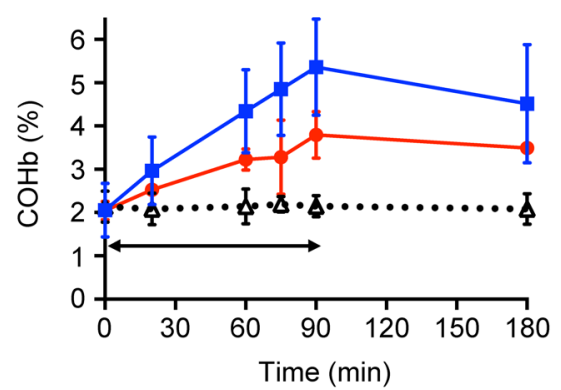

E

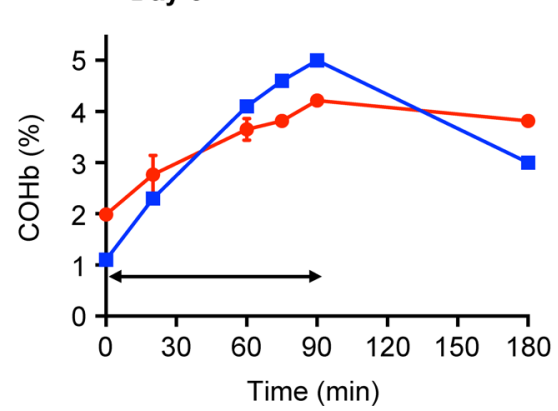

C $\begin{array}{ll} & p<0.0001 \text { time } \\ & p=0.0168 \text { treatment } \\ & p<0.0001 \text { interaction }\end{array}$

Figure 3. Mean carboxyhemoglobin levels in participants treated with iCO versus placebo air on days 1-5. Arterial blood was drawn from participants for measurement of baseline carboxyhemoglobin $(\mathrm{COHb})$ level prior to treatment. If baseline $\mathrm{COHb}$ was $<3 \%$, subjects were treated with medical-grade air or iCO for 90 minutes. Arterial blood was drawn for $\mathrm{COHb}$ monitoring at 20,60,75, and 90 minutes. Additional blood was drawn for measurement of $\mathrm{COHb}$ levels at 180 minutes (90 minutes after completion of treatment). (A-E) COHb levels for iCO-treated and placebo-treated participants on each day of treatment. COHb levels were measured in triplicate for each subject at each time point and mean value obtained. (A) Day 1, $n=4 \mathrm{iCO} 100 \mathrm{ppm}, n=2 \mathrm{iCO} 200$ ppm, $n=4$ placebo. (B) Day 2, $n=4$ iCO 100 ppm, $n=4$ iCO 200 ppm, $n=4$ placebo. (C) Day 3, $n=2$ iCO 100 ppm, $n=2$ iCO 200 ppm, $n=2$ placebo. (D) Day 4, $n=4$ iCO 200 ppm, $n=1$ placebo. (E) Day 5, $n=2$ iCO 100 ppm, $n=1 \mathrm{iCO} 200$ ppm. Data are mean and SD for each group on each day of treatment. COHb levels were significantly different between treatment groups and over time on days 1-3 by 2-way ANOVA followed by Tukey's post hoc test (Supplemental Table 6). Arrows indicate duration of treatment.

No significant differences were observed in baseline (Supplemental Figure 5, B and C) or post-treatment (Figure 6, B and C, and Supplemental Figures 8 and 9) RIPK3 or IL-18 levels between iCO-treated and placebo-treated patients. A panel of cytokine and chemokine exploratory biomarkers including IL-6, IL-8, IL-10, IL-1Ra, and IL-1 $\beta$ (Supplemental Table 8) were analyzed by multiplex assay using Luminex, none of which were significantly different among treatment groups at baseline or days 1-5 (data not shown).

\section{Discussion}

This is the first study to our knowledge to examine inhalation of low-dose iCO as a potential new therapy in critically ill patients. We show that precise administration of low-dose iCO using a ventilator-compatible $\mathrm{CO}$ delivery system is feasible in mechanically ventilated patients with sepsis-induced ARDS. Low-dose iCO was well tolerated in 8 patients with moderate to severe ARDS, with no evidence of administrationassociated AEs and no $\mathrm{COHb}$ levels that exceeded $10 \%$. None of the reported SAEs were judged to be related to iCO or study procedures. The CFK equation was highly accurate at predicting $\mathrm{COHb}$ levels, even in the setting of moderate to severe ARDS, based on the COHb level measured after 20 minutes of 200 ppm CO administration. This represents what we believe to be a novel application of the CFK equation, and demonstrates that iCO therapy can be effectively delivered with real-time monitoring of measured and predicted $\mathrm{COHb}$ levels to ensure consistent systemic uptake in ARDS patients with impaired gas exchange. While additional studies are necessary to confirm the safety of iCO in a larger cohort of patients, the strong correlation $(r=0.916)$ and accuracy $\left(R^{2}=0.9204\right)$ to predict COHb levels using the $\mathrm{CFK}$ equation should ensure that $\mathrm{COHb}$ levels remain in a safe target range during future trials. 
Table 4. Serious adverse events by participant

\begin{tabular}{|c|c|c|c|}
\hline Subject & Treatment & Adverse event & Description \\
\hline Subject 2 & Air & $\begin{array}{l}\text { Compartment syndrome, knee } \\
\text { disarticulation on day } 4\end{array}$ & $\begin{array}{l}\text { Patient developed right leg compartment syndrome requiring a fasciotomy prior to } \\
\text { enrollment; required right knee disarticulation on study day } 4\end{array}$ \\
\hline Subject 2 & Air & Tracheostomy on day 10 & $\begin{array}{l}\text { Patient required tracheostomy for persistent respiratory failure on study day } 10 \text {; } \\
\text { was decannulated on study day } 40\end{array}$ \\
\hline Subject 3 & CO & $\begin{array}{l}\text { Laryngeal edema and } \\
\text { tracheostomy on day } 7\end{array}$ & $\begin{array}{l}\text { Patient had history of laryngeal cancer requiring radiation and a difficult intubation } \\
\text { due to airway edema; developed upper airway edema following extubation requiring a } \\
\text { tracheostomy on study day } 7 \text {; transitioned to unassisted breathing on study day } 9\end{array}$ \\
\hline Subject 6 & Air & $\begin{array}{l}\text { Respiratory failure, tracheostomy } \\
\text { on day } 11\end{array}$ & $\begin{array}{l}\text { Due to persistent hypoxemia and ongoing need for mechanical ventilation, patient } \\
\text { underwent a tracheostomy on study day } 11\end{array}$ \\
\hline Subject 9 & CO & $\begin{array}{l}\text { Respiratory failure, tracheostomy } \\
\text { on day } 8\end{array}$ & $\begin{array}{l}\text { Patient required tracheostomy placement on study day } 8 \text { for persistent ventilator } \\
\text { dependence secondary to ARDS, to facilitate further ventilator weaning; transitioned to } \\
\text { unassisted breathing on study day } 15 \text { and was decannulated on study day } 26\end{array}$ \\
\hline
\end{tabular}

LAR, legally authorized representative.

In this study, we show that low-dose iCO therapy can be safely and effectively administered in mechanically ventilated ARDS patients in an intensive care unit (ICU) setting using a ventilator-compatible CO delivery system. Low-dose iCO significantly increased $\mathrm{COHb}$ levels in a dose-dependent fashion, with mean $\mathrm{COHb}$ levels of $3.48 \%$ and $4.9 \%$, and maximal increases to $4.4 \%$ and $6.87 \%$, in $100 \mathrm{ppm}$ and $200 \mathrm{ppm}$ iCO-treated ARDS subjects, respectively. In our prior work in a baboon pneumonia-induced ALI model, we achieved a target $\mathrm{COHb}$ level of $6 \%-8 \%$ after $200 \mathrm{ppm}$ iCO treatment for 60 minutes (9). Although $\mathrm{COHb}$ levels were higher in nonhuman primates with ALI compared with ARDS patients in this phase I trial, these results were predictable, based on key variables in the CFK equation that influence CO uptake.

Table 5. Secondary respiratory and systemic outcomes by subject

\begin{tabular}{|c|c|c|c|c|c|c|c|c|c|}
\hline Subject & Treatment & LIS (day 7) & SOFA (day 7) & $\begin{array}{l}\text { Duration of } \\
\text { mechanical } \\
\text { ventilation } \\
\text { (days) }\end{array}$ & $\begin{array}{l}\text { Ventilator-free } \\
\text { days (days 1-28) }\end{array}$ & $\begin{array}{l}\text { Duration of } \\
\text { vasopressors } \\
\text { (days) }\end{array}$ & $\begin{array}{l}\text { Vasopressor- } \\
\text { free days } \\
\text { (days 1-28) }\end{array}$ & $\begin{array}{l}\text { ICU-free days } \\
\text { (days 1-28) }\end{array}$ & $\begin{array}{l}\text { Vital status } \\
\text { and day of } \\
\text { discharge }\end{array}$ \\
\hline Subject 1 & CO & 1.33 & 3 & 8 & 21 & 5 & 23 & 18 & Alive, day 10 \\
\hline Subject 2 & Air & 2.5 & 12 & 14 & 17 & 16 & 14 & 0 & Alive, day 71 \\
\hline Subject 3 & CO & 2.33 & 3 & 9 & 20 & 6 & 23 & 13 & Alive, day 14 \\
\hline Subject 6 & Air & 3.33 & 17 & 32 & 0 & 16 & 12 & 0 & Alive, day 33 \\
\hline \multicolumn{10}{|l|}{ Cohort 2} \\
\hline Subject 7 & Air & 1.33 & 0 & 6 & 24 & 6 & 24 & 23 & Alive, day 11 \\
\hline Subject 8 & CO & 2.0 & 7 & 14 & 18 & 2 & 26 & 17 & Alive, day 14 \\
\hline Subject 9 & CO & 2.25 & 10 & 31 & 1 & 4 & 25 & 9 & Alive, day 24 \\
\hline
\end{tabular}

LIS, lung injury score; SOFA, sequential organ failure assessment. 
A

iCO 100 ppm (Cohort 1)

$r=0.8614, p<0.0001$

$R^{2}=0.7186, p<0.0001$

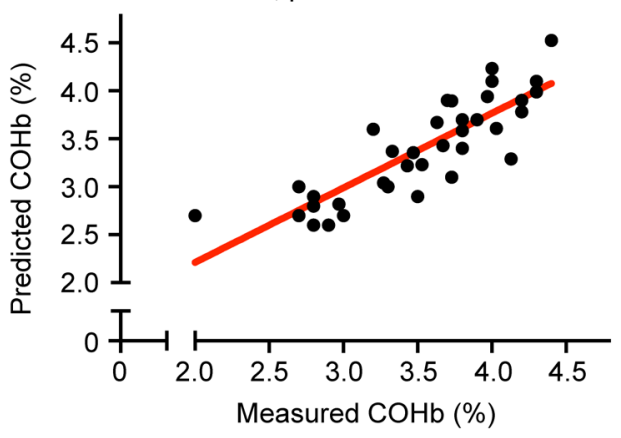

B

iCO 200 ppm (Cohort 2)

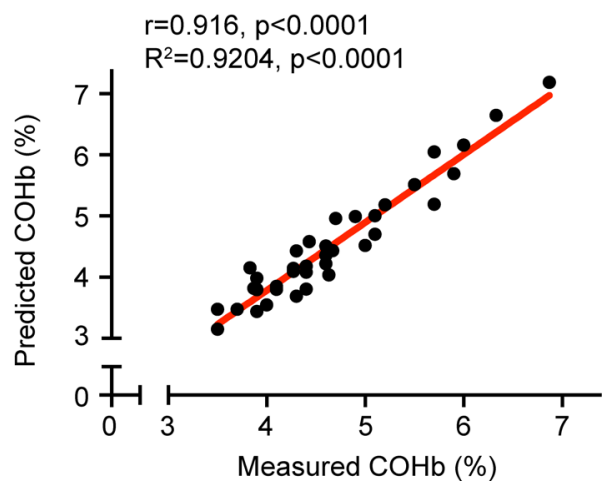

C

iCO 100 ppm (Cohort 1)

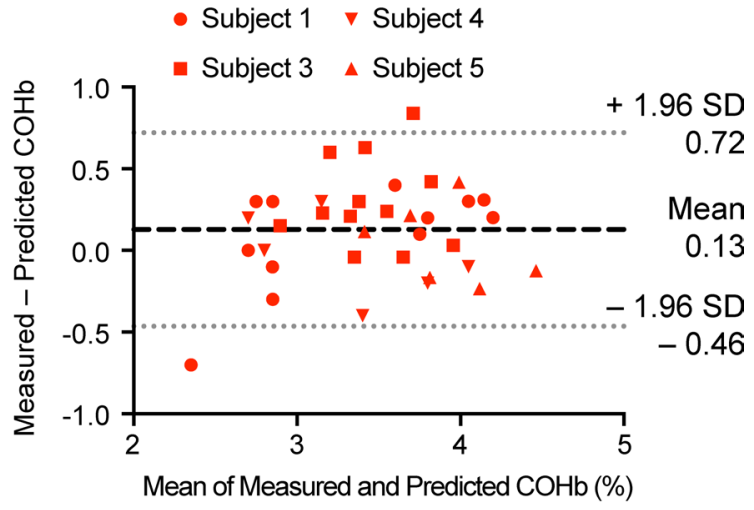

D

iCO 200 ppm (Cohort 2)

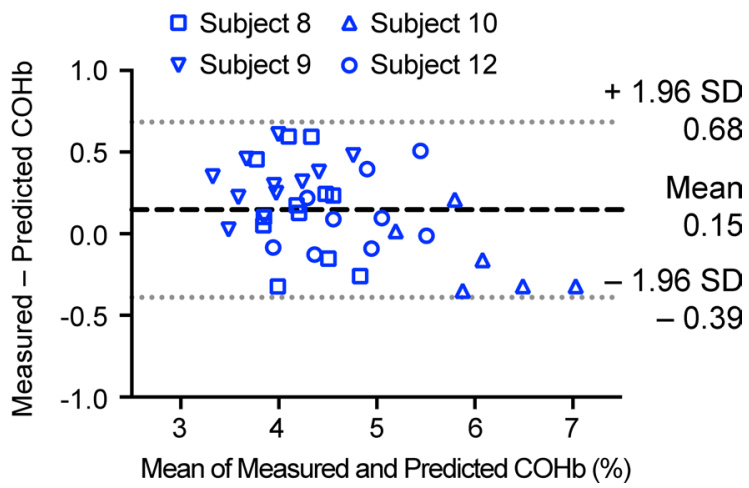

Figure 4. Coburn-Forster-Kane equation accurately predicts COHb levels in ARDS patients. The Coburn-Forster-Kane (CFK) equation was used to predict COHb levels at 60,75, and 90 minutes using the measured $\mathrm{COHb}$ level at baseline and 20 minutes in subjects treated with $100 \mathrm{ppm}$ (day $1, n=4 ;$ day $2, n=$ 4; day 3, $n=2$; day 5, $n=2$ ) (A and C) or 200 ppm (day 1, $n=2$; day 2, $n=4$; day 3, $n=2$; day 4, $n=4$; day 5, $n=1$ ) (B and D) iCO. Accuracy of the CFK equation in predicting $\mathrm{COHb}$ levels was analyzed by Spearman's correlation and Bland-Altman plots using measured vs. predicted COHb levels and modeled using linear regression. (A) Correlation between predicted and measured COHb levels using the 20-minute COHb level and CFK equation in 100 ppm iCO-treated subjects in cohort 1 (Spearman's $r=0.8614, P<0.0001$; goodness-of-fit $R^{2}=0.7186, P<0.0001$ ). (B) Correlation between predicted and measured COHb levels using the 20-minute COHb level and CFK equation in 200 ppm iCO-treated subjects in cohort 2 (Spearman's $r=0.916, P<0.0001$; goodness-of-fit $R^{2}=0.9204, P<0.0001$ ). (C and D) Bland-Altman plots demonstrate excellent agreement between measured and predicted COHb levels in $100 \mathrm{ppm}$ iCOtreated subjects in cohort 1 (C) and 200 ppm iCO-treated subjects in cohort 2 (D).

In addition to lower weight, which affects blood volume, the baboons in our prior study had significant differences in alveolar ventilation, $\mathrm{FiO}_{2}$, and $\mathrm{DL}_{\mathrm{CO}}$, which together accounted for the increased $\mathrm{CO}$ uptake compared with ARDS subjects in this trial.

In this study, we also found that there was a trend toward improved respiratory and systemic secondary endpoints in iCO-treated patients, particularly the LIS and SOFA scores on day 7. SOFA scores declined to the greatest extent in iCO-treated subjects in cohort 1 compared with iCO-treated subjects in cohort 2 or placebo-treated subjects, although this was not powered for statistical significance. We did find a statistically significant difference in plasma mtDNA levels between $\mathrm{iCO}$-treated and placebo-treated subjects. CO-treated patients had a significant reduction in mtDNA levels on day 2 compared with pretreatment baseline levels versus the respective mtDNA changes in placebo-treated subjects. This reached statistical significance on day 2 in both cohorts and on day 4 in cohort 2, with similar trends on other days, although the majority of subjects did not receive treatment beyond study day 3 or 4 . Furthermore, 2 subjects were excluded from the mtDNA analysis, which limits the robustness of our finding. IL-18 and RIPK3 levels did not differ among the treatment groups, and an exploratory Luminex biomarker panel of 41 cytokines and chemokines showed no significant differences between iCO- and placebo-treated subjects.

Evidence suggests that mitochondrial dysfunction, including release of mtDNA, plays a key role in the pathogenesis of sepsis and multiple organ dysfunction $(38,40-43)$. Moreover, early activation of 

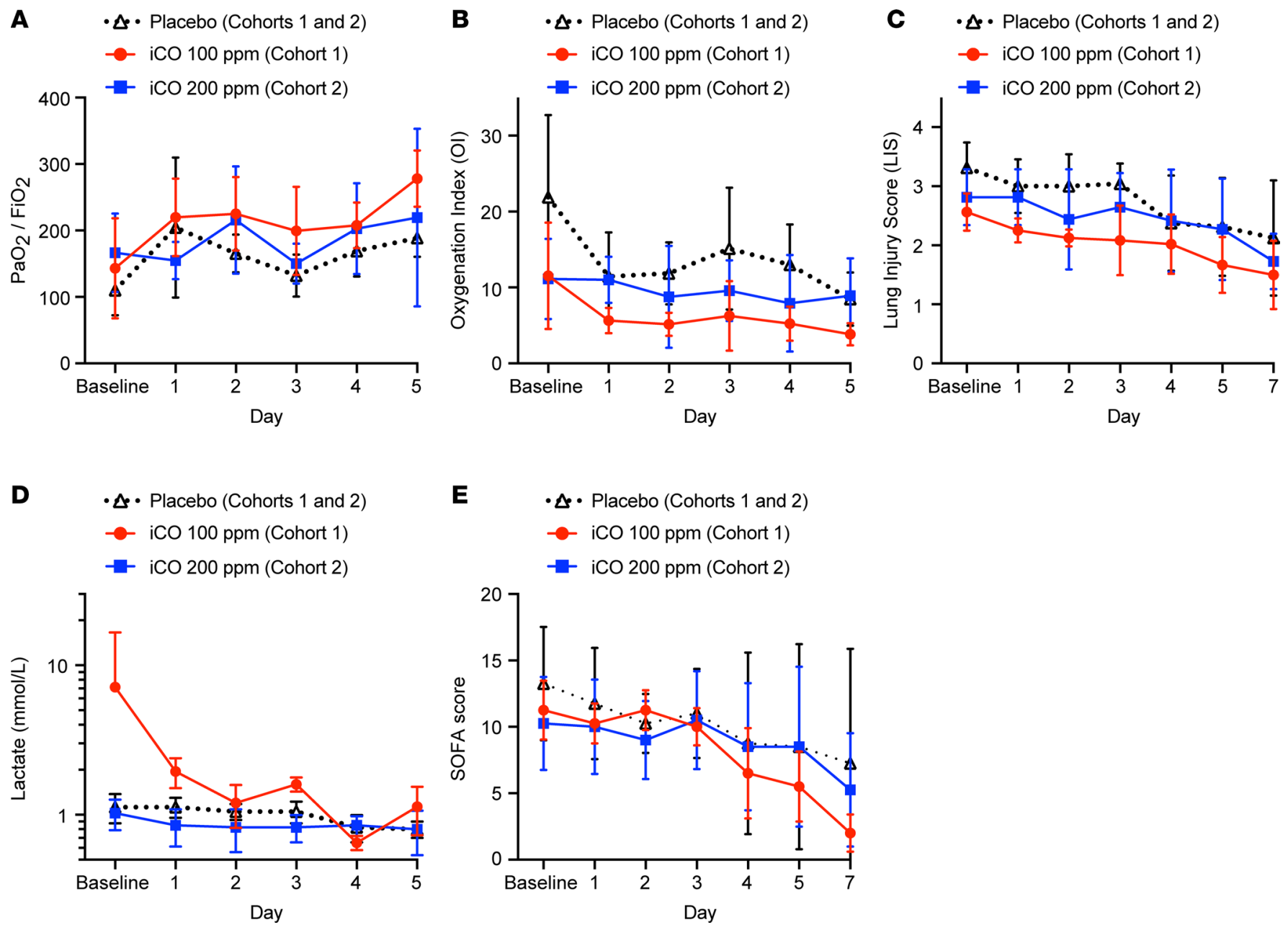

Figure 5. Secondary respiratory and systemic outcomes. Mean and SD for (A) $\mathrm{PaO}_{2} / \mathrm{FiO}_{2}$, (B) oxygenation index (OI), (C) lung injury score (LIS), (D) lactate, and (E) sequential organ failure assessment (SOFA) score for subjects treated with placebo air $(n=4)$, iCO 100 ppm $(n=4)$, or iCO 200 ppm $(n=4)$ at baseline, study days $1,2,3,4,5$, and 7 .

mitochondrial biogenesis has been associated with improved survival in critically ill patients with sepsis (44). Sepsis-induced mitochondrial dysfunction can lead to release of mtDNA into the circulation during critical illness (38), where it acts as a damage-associated molecular pattern (DAMP) to activate innate immune responses and cell death pathways $(38,41,43,45)$. We and others have demonstrated that circulating mtDNA levels are significantly increased in patients with sepsis and ARDS, and correlate with mortality in critically ill patients $(38,40,42)$. Importantly, we also have shown that CO can inhibit mitochondrial ROS generation, inhibit translocation of mtDNA into the cytosol, and preserve mitochondrial function in macrophages (16). CO has also been shown to activate mitochondrial biogenesis in skeletal muscle in humans (25). Although limited by the small patient numbers in this phase I trial, our mtDNA results are provocative and suggest that iCO therapy may reduce circulating mtDNA levels, perhaps by protecting mitochondria, which could in turn lead to beneficial clinical outcomes. Future efficacy studies are needed to validate these results in a larger patient population and determine whether these biological marker findings correlate with clinical outcomes in patients with sepsis-induced ARDS.

Our phase I trial had inherent limitations due to the small number of participants. This limits our conclusions about secondary outcomes, as no subjects completed 5 days of study drug treatment. As the primary endpoint of this phase I study was safety, we believed it was necessary to establish stringent criteria for daily treatment in this vulnerable population of critically ill patients. Study drug holds on the basis of anemia, severe hypoxemia, and lack of an arterial line for monitoring were based on predefined safety criteria in this first-in-human CO ARDS study. Furthermore, the most common reason for holding treatment 
A 1000

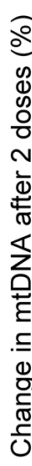

政

600
400
200
100
80
60
40
20

00
00
80
60
40
20

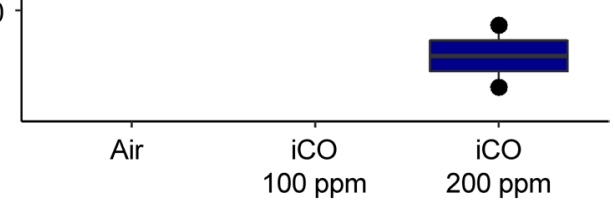

B

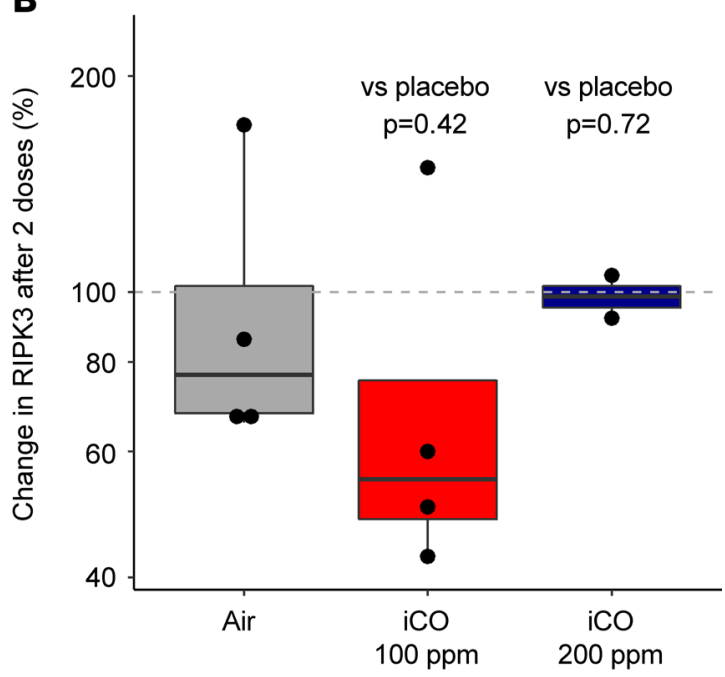

C

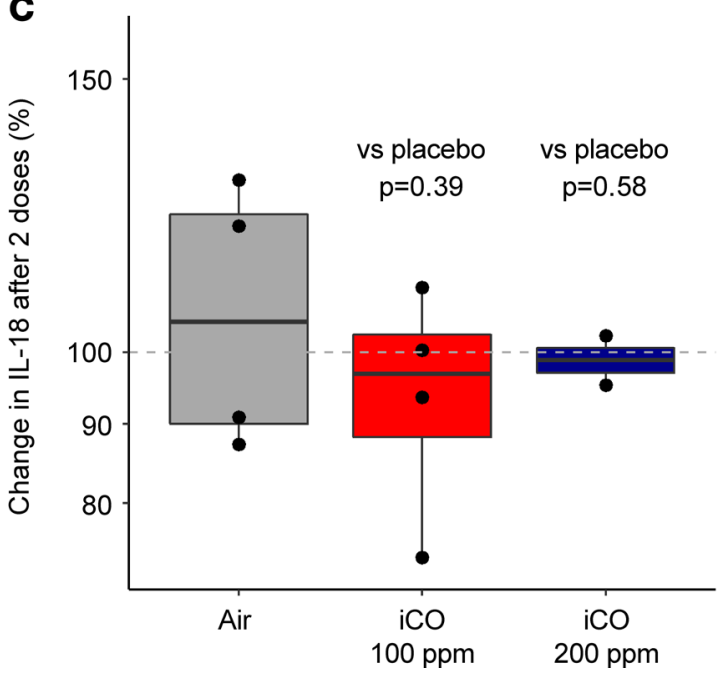

Figure 6. Circulating mtDNA levels are decreased in iCO-treated subjects compared with placebotreated subjects. Plasma levels of (A) mtDNA, (B) RIPK3, and (C) IL-18 were measured in subjects before treatment on day 1 and after treatment on day 2 in subjects $(n=10)$ completing 2 days of treatment. Mean changes in mtDNA, RIPK3, and IL-18 in iCO-treated subjects (100 ppm and 200 ppm) were compared with changes in placebo-treated subjects in a pairwise manner using $t$ tests. Box plots show 25 th, median, and 75 th percentiles.

was discontinuation of mechanical ventilation, which was a prespecified study drug completion endpoint of the trial. In addition, the primary safety endpoint analysis was prespecified to include all subjects who completed at least one dose of the study drug. Moreover, the feasibility of iCO administration in this study was based on the successful completion of at least one dose of the study drug in mechanically ventilated patients with sepsis-induced ARDS. 
Although trends in secondary respiratory and systemic outcomes were observed, we did not have adequate power to observe significant differences in major clinical outcomes. While we found a statistically significant difference in mtDNA levels in iCO-versus placebo-treated patients, we did not find differences in any other biomarkers, which may be due to the small sample size or sampling at an incorrect time point. It is also possible that differences in baseline severity of illness confounded secondary clinical and biomarker outcomes, although we attempted to account for this by analyzing within-patient differences. Another limitation, as with many critical care trials, is distinguishing clinical outcomes associated with participants' underlying critical illness versus those from the study intervention.

Our first-in-human ARDS iCO study has several notable strengths. The rigorous safety testing of iCO using the CO delivery system and validation of the CFK equation in mechanically ventilated ARDS patients significantly advances iCO as a therapeutic. This study not only established key technical and physiologic platforms to safely and accurately administer iCO in critically ill patients, but also advances translation of iCO therapy in other diseases where iCO holds substantial promise $(46,47)$. While we found that low doses of 100 and 200 ppm CO were safe in a small group of mechanically ventilated ARDS patients, we also found that patients with ARDS of varying severity have different degrees of CO uptake with fixed iCO dosing based on the variables in the CFK equation. As the CFK equation is highly accurate at predicting $\mathrm{COHb}$ levels, our findings suggest that the CFK equation can be used to individually titrate $\mathrm{iCO}$ dosing to achieve a target $\mathrm{COHb}$ level in ARDS patients with varying degrees of impaired gas exchange. Future studies are necessary to evaluate the optimal duration of iCO treatment, confirm safety in a larger patient population, and evaluate the utility of a CFK equation-based iCO dosing algorithm to safely and accurately administer low-dose iCO to patients with ARDS.

\section{Methods}

Study design. We conducted a multicenter, randomized, placebo-controlled, dose-escalation phase I clinical trial of low-dose iCO in patients with sepsis-induced ARDS. The study was designed to enroll 12 patients sequentially in 2 fixed-dose iCO cohorts: 6 patients in cohort 1 (4 CO 100 ppm; 2 placebo) followed by a safety data review and then 6 additional patients in cohort 2 (4 CO 200 ppm; 2 placebo). The dose selection and study design of 6 patients randomized in a 2:1 ratio in each cohort were based on FDA guidance to mitigate risk in first-in-human trials $(48,49)$.

The trial was designed for subjects to receive the study drug for 90 minutes once daily for at least 1 day, but up to 5 consecutive days. We selected once-daily iCO treatment based on prior work demonstrating both safety and activation of mitochondrial biogenesis in healthy volunteers after once-daily iCO treatment (200 ppm for 60 minutes) on 5 consecutive days $(17,25)$. Preclinical studies have shown that CO treatment for short periods of time induces transcriptional programs of mitochondrial biogenesis $(9,17,50,51)$ and induces autophagy $(11,15,52)$, suggesting that once-daily dosing of $\mathrm{CO}$ could be effective, in contrast to continuous administration, as with NO. Once-daily iCO dosing also optimizes subject safety by ensuring that CO accumulation over sequential days will not occur as treatments are separated by 4-6 half-lives for CO elimination.

Ninety minutes of treatment was chosen based on our prior studies in nonhuman primates (9) showing that iCO treatment at 200 ppm for 90 minutes is safe and does not lead to $\mathrm{COHb}$ levels exceeding $10 \%$. We also used the $\mathrm{CFK}$ equation to predict $\mathrm{COHb}$ levels for a range of treatment times and values for diffusing capacity of the lung for $\mathrm{CO}\left(\mathrm{DL}_{\mathrm{CO}}\right)$, alveolar ventilation $\left(\mathrm{V}_{\mathrm{A}}\right)$, weight, $\mathrm{Hgb}$, and $\mathrm{FiO}_{2}$ in patients with ARDS. This modeling showed that $\mathrm{COHb}$ levels are unlikely to exceed $10 \%$ in ARDS subjects after 90 minutes of treatment with 200 ppm. Longer durations of treatment could increase $\mathrm{COHb}$ levels beyond $10 \%$, which could lead to adverse effects in this critically ill patient population with impaired gas exchange.

Patients were enrolled in cohorts 1 and 2 in the ICUs at BWH, MGH, and Duke University Hospital, between July 10, 2015, and November 14, 2016. Patients were also screened in the ICU at New York-Presbyterian Hospital/Weill Cornell Medical Center, but no participants were enrolled. A CFK algorithm-determined iCO dose cohort (cohort 3) was initially planned, but funding for the trial ended before participants could be enrolled in cohort 3. The study protocol can be found in the supplemental material available online with this article.

Participants. Adult patients $\geq 18$ years old were eligible for inclusion if they met criteria for sepsis and ARDS according to consensus criteria (Supplemental Table 9) $(53,54)$. Sepsis was initially defined as systemic inflammatory response syndrome (SIRS) plus suspected or documented infection $(55,56)$; however, the definition was later modified to reflect new consensus guidelines, namely suspected or documented 
infection and a SOFA score $\geq 2$ (54). ARDS was defined as the acute onset of (i) bilateral infiltrates consistent with pulmonary edema on frontal chest radiograph; (ii) a $\mathrm{PaO}_{2} / \mathrm{FiO}_{2}$ ratio $\leq 300$, with at least $5 \mathrm{~cm}$ $\mathrm{H}_{2} \mathrm{O}$ positive end-expiratory airway pressure (PEEP); (iii) a need for positive pressure ventilation by an endotracheal or tracheal tube; and (iv) no clinical evidence of left atrial hypertension that could account for bilateral pulmonary infiltrates (53). All enrolled participants had suspected or documented infection, were treated with antibiotics, met SIRS criteria, and had baseline SOFA scores $\geq 2$, thereby meeting both the sepsis-1 and sepsis-3 definitions of sepsis (54-56).

Patients with severe hypoxemia, recent cardiac disease, or stroke were excluded due to the risk of inadequate oxygen delivery. Patients on inhaled pulmonary vasodilators were excluded, as these could interfere with CO delivery. Patients were also excluded if they had New York Heart Association (NYHA) class IV cardiopulmonary disease or were moribund, or there was no commitment to full supportive measures. Active cigarette smokers were initially excluded from participation, but this criterion was eliminated, as smoking history from surrogate decision-makers was thought to be unreliable, and pre-admission $\mathrm{COHb}$ levels would have normalized, as the average $\mathrm{COHb}$ half-life is 240 minutes on $21 \%$ oxygen (33) and shorter on supplemental oxygen. Moreover, the study drug would be held if daily $\mathrm{COHb}$ was $\geq 3 \%$. Patients with $\mathrm{Hgb} \leq 8 \mathrm{~g} / \mathrm{dl}$ or $\mathrm{Hgb} \leq 9$ and actively bleeding were also initially excluded, but this criterion was later modified (to $\mathrm{Hgb} \leq 7.5$ or $\leq 8 \mathrm{~g} / \mathrm{dl}$, respectively) to reflect the current restrictive transfusion strategy in critically ill patients (57).

Additional eligibility criteria were modified based on the DSMB recommendations, including: (i) elimination of SIRS inclusion criteria to align with sepsis-3 definitions (54); (ii) increased allowance of time frame for ARDS onset from 96 to 120 hours; (iii) elimination of chronic respiratory failure as exclusion, and modification of exclusion \#17 from NYHA class IV cardiac disease to NYHA class IV cardiopulmonary disease, which ensured that patients with chronic respiratory failure and poor functional class were excluded from enrollment; (iv) elimination of the severe chronic liver disease exclusion, as chronic liver disease patients with high morbidity and mortality were already excluded by criterion 8 (moribund patient not expected to survive 24 hours). Supplemental Table 9 shows the full inclusion and exclusion criteria.

Randomization and masking. Participants were randomized to receive iCO or placebo air at a ratio of 2:1 using a permuted block method (block size of 3) (58). We used SAS version 9.4 to generate the sequence to assign participants to treatment arms, which was prepared in advance by the study statistician and provided to the DCC physicians. Following receipt of written informed consent from eligible patients or their LAR or surrogate, site investigators contacted an unmasked DCC physician member to request a randomization number and study assignment from the preprepared randomization schedule. The study coordinators and clinical staff at each site were masked to treatment assignments. The respiratory therapist (RT) and physician investigator administering the study drug were unmasked to treatment groups given the safety monitoring required in this phase I trial. In cohort 2, based on a DSMB recommendation to ensure blinding of block assignment, investigators were masked to study drug assignments for subjects enrolled at other sites, but remained unmasked to site-specific participant allocation. To maintain masking at each site, subjects randomized to placebo were administered medical-grade air in the same fashion as iCO-randomized subjects and from identical-appearing gas cylinders. The unmasked RT and physician investigator concealed the gas cylinders, $\mathrm{CO}$ delivery system, and CO-related measurements to ensure that the study coordinator and clinical staff remained blinded to the study drug assignment. The unmasked site investigators entered CO-related measurements into electronic case report forms within the password-protected database, but the masked research coordinators did not have access to these data.

Procedures. CO or placebo air was administered via inhalation to subjects via COventDS (12th Man Technologies), a delivery system approved as part of our investigational new drug (IND, no. 122800) to administer $\mathrm{CO}$ through a mechanical ventilator (9). The source gas tanks contained either 5,000 ppm $(0.5 \%) \mathrm{CO}$ in medical-grade air or placebo (medical- grade air) (Praxair Healthcare Services). The source gas was injected into the ventilator circuit in proportion to the gas flow to provide a constant delivered dose, which was monitored in real time by a calibrated sidestream electrochemical analyzer (9).

Subjects had blood drawn daily prior to study drug administration for measurement of $\mathrm{COHb}$ and lactate levels. If $\mathrm{COHb}$ was $\geq 3 \%$ or lactate $\geq 4 \mathrm{mmol} / 1$, the study drug was held, and the subject was reevaluated for treatment the next day. An ECG was performed daily prior to study drug administration to assess cardiac exclusion criteria, including ST elevation, myocardial infarction (MI), or unstable angina/ non-ST elevation MI concerning for acute coronary syndrome. The study drug was also held if a subject 
developed (i) severe hypoxemia defined as an oxygen saturation level measured by pulse oximetry $\left(\mathrm{SpO}_{2}\right)$ $<95 \%$ or $\mathrm{PaO}_{2}<80 \mathrm{mmHg}$ on $\mathrm{FiO}_{2} \geq 0.8$; (ii) $\mathrm{Hgb}<7.5 \mathrm{~g} / \mathrm{dl}$ or $\mathrm{Hgb}<8 \mathrm{~g} / \mathrm{dl}$ and active bleeding; (iii) diffuse alveolar hemorrhage from vasculitis; (iv) use of high-frequency ventilation; or (v) use of inhaled pulmonary vasodilator therapy (e.g., NO or prostaglandins).

Following randomization, subjects were administered the study drug for 90 minutes once daily for up to 5 consecutive days or until mechanical ventilation was discontinued, whichever occurred first. All participants who received at least one dose of the study drug were included in the analysis.

The study drug concentration was monitored continuously with the COventDS built-in analyzer to ensure that the dose was delivered accurately. As a safety feature, the COventDS has an internal and microprocessor-independent gas analyzer of inspired gases with alarms that sound on high or low $\mathrm{CO}$ or $\mathrm{O}_{2}$ concentrations. Participants were continuously monitored noninvasively (SpCO) using a Masimo Radical 7 (Rad-7) pulse oximeter (Masimo). Ambient air CO concentrations were measured continuously with a Pac $7000 \mathrm{CO}$ detector (Dräger) to ensure that ambient levels were maintained within the recommended limits for occupational exposure (9). Arterial blood was drawn prior to dosing and at 20, 60, 75, 90, and 180 minutes for safety monitoring of COHb levels using an IL682 CO-oximeter (9) (Instrumentation Laboratories), and for arterial blood gas analysis. The baseline and 20-minute $\mathrm{COHb}$ levels were used in a MATLAB-generated (MathWorks) CFK calculator program to estimate $\mathrm{DL}_{\mathrm{CO}}$ and compute the predicted COHb level at 90 minutes. A physician investigator and RT remained at the bedside for the duration of the treatment and were immediately available for an additional 90 minutes afterward.

To assure safety, the following study drug holds were implemented. During study drug administration, iCO would be stopped prior to 90 minutes if the measured $\mathrm{COHb}$ was $>7 \%$. If the $\mathrm{COHb}$ was predicted to be $>7 \%$ by the CFK equation prior to 90 minutes, the study drug would be stopped at that time, and an arterial $\mathrm{COHb}$ would be measured. Prespecified administration-associated AEs were defined based on discussions with the DSMB and are shown in Supplemental Table 10. The study drug was permanently discontinued if a subject had a (i) prespecified administration-associated AE; (ii) SAE related to the study drug; (iii) 3 or more missed doses due to $\mathrm{COHb}$ levels $\geq 3 \%$; or (iv) if the investigator, attending physician, subject, or their surrogate decided to stop.

We also assessed the LIS and SOFA score. The LIS has been shown to correlate with lung physiology, mortality, and ventilator-free days (VFDs) (59-61), and SOFA score has been shown to be a reliable prognostic indicator of outcomes in critically ill patients (34). The LIS is a composite 4-point scoring system including $\mathrm{PaO}_{2} / \mathrm{FiO}_{2}, \mathrm{PEEP}$, quasi-static respiratory compliance (when available), and the extent of infiltrates on chest X-ray (62). If a patient was no longer ventilated, PEEP was assigned as $\leq 5 \mathrm{~cm} \mathrm{H}_{2} \mathrm{O}$ and $\mathrm{PaO}_{2} / \mathrm{FiO}_{2}$ as $\geq 300 \mathrm{mmHg}$, and the compliance was not calculated; therefore the number of points was divided by 3 instead of 4 (63). When a chest X-ray was not available on a given day, the prior day's chest $\mathrm{X}$-ray score was used in the LIS calculation. For the SOFA score calculation, if a value was missing, we carried forward the value from the prior measurement. For LIS and SOFA score calculations, when $\mathrm{PaO}_{2} /$ $\mathrm{FiO}_{2}$ was not available, the $\mathrm{PaO}_{2}$ was estimated from the $\mathrm{SpO}_{2}$ using nonlinear imputation, and the $\mathrm{SpO}_{2} /$ $\mathrm{FiO}_{2}$ equivalent was used as described previously $(64,65)$. For the cardiovascular dysfunction component of the SOFA score, patients on phenylephrine and vasopressin were scored using estimated norepinephrine equivalences based on prior studies $(66,67)$. Subjects who no longer required mechanical ventilation were scored 0 for the respiratory component of the SOFA score, as the $\mathrm{PaO}_{2} / \mathrm{FiO}_{2}$ ratio was either unavailable or could not be reliably compared with the $\mathrm{PaO}_{2} / \mathrm{FiO}_{2}$ ratio in mechanically ventilated subjects. $\mathrm{SpO}_{2} / \mathrm{FiO}_{2}$ was not used to impute the $\mathrm{PaO}_{2} / \mathrm{FiO}_{2}$ ratio in subjects no longer requiring mechanical ventilation, as this methodology has only been validated in mechanically ventilated ARDS patients $(64,65)$.

We also measured biological markers in the plasma, collected before treatment and 90 minutes after treatment, on days 1-5. If no treatment was given, biomarkers were measured daily. In a subgroup of patients enrolled at BWH, discarded plasma samples were also obtained from the clinical laboratories on study day 7. mtDNA was measured by quantitative PCR of human NADH dehydrogenase 1 (MTND1) (38). IL-18 levels were measured using a human IL-18 ELISA kit (37) (R\&D Systems), and RIPK3 levels were measured using a human RIPK3 ELISA kit (68) (CUSABIO Technology). Levels of cell-free mtDNA were measured in plasma as previously described (38). Briefly, $50 \mu 1$ plasma was mixed with $170 \mu 1$ PBS, followed by brief vortexing. The diluted plasma was centrifuged at $700 \mathrm{~g}$ at $4^{\circ} \mathrm{C}$ for 5 minutes, and the supernatant $(200 \mu \mathrm{l})$ was saved by avoiding touching any pellets and the bottom of the tubes with pipette tips. The obtained supernatant was further centrifuged at $18,000 \mathrm{~g}$ at $4^{\circ} \mathrm{C}$ for 15 minutes, and the resulting 
supernatant $(180 \mu \mathrm{l})$ was carefully saved. DNA was isolated from the supernatant using the DNeasy Blood and Tissue Kit (QIAGEN) according to the manufacturer's protocol. DNA levels were measured in diluted samples using SYBR Green dye-based quantitative PCR using a PRISM 7300 sequence detection system (Applied Biosystems). Primer sequences (38) were as follows: human MTND1 gene: forward 5-ATACCCATGGCCAACCTCCT-3, reverse 5-GGGCCTTTGCGTAGTTGTAT-3. Plasmid DNA with complementary DNA sequences for human MTND1 was obtained from OriGene SC101172).

A panel of additional cytokines/chemokines was measured by multiplex assay (HCYTMAG-60K-PX41, MilliporeSigma) using Luminex.

After each cohort had completed enrollment, there was a study pause for a review of the data by the DSMB. The DSMB consisted of 3 critical care physicians with expertise in ARDS and critical care trials. In addition, a medical monitor and an SRC, consisting of the study investigators at each site, evaluated the safety data at the end of each cohort and made recommendations to the DSMB about proceeding with enrollment.

Outcomes. Because this is the first trial to our knowledge to administer iCO to mechanically ventilated patients with ARDS, the primary endpoint of this phase I trial was feasibility and safety of iCO administration in this critically ill patient population. The primary safety outcome was defined as the incidence of prespecified administration-associated AEs and SAEs, with a safety analysis focused on the incidence of $\mathrm{COHb}$ level $\geq 10 \%$ in study participants. All AEs and SAEs were recorded during the period from enrollment to ICU discharge. We classified all AEs and SAEs by system organ class, and AE severity was graded according to the CTCAE version 4 grading scale. A subset of AEs were considered "administration-related AEs" (Supplemental Table 10) and were by definition considered suspected adverse reactions. All AEs were reported to the DCC, DSMB, FDA, and site IRBs according to local and protocol-specified timelines, including prior to commencing enrollment in cohort 2.

The secondary endpoint was the accuracy of iCO dosing as determined by the CFK equation $(22,69)$ (Supplemental Figure 10) to predict $\mathrm{COHb}$ levels. We evaluated the accuracy of the CFK equation in predicting $\mathrm{COHb}$ levels at 60, 75, and 90 minutes in iCO-treated participants in cohorts 1 and 2 . The variables in the CFK equation were calculated as described previously (22). $\mathrm{CO}$ production $\left(\mathrm{V}_{\mathrm{CO}}\right)$ was assumed to be $0.007 \mathrm{ml} / \mathrm{min}$, and the affinity constant $M$ was assumed to be 218 (22). To calculate alveolar ventilation, a NICO monitor (Philips Respironics) was used to measure exhaled $\mathrm{CO}_{2}$ and calculate dead space using the Enghoff modification of the Bohr equation. Alveolar ventilation was calculated as the difference between minute ventilation and dead space ventilation. To estimate $\mathrm{DL}_{\mathrm{CO}}$, we input the baseline $\mathrm{COHb}$ level (time $=$ 0 ) and the $\mathrm{COHb}$ level measured after 20 minutes of iCO treatment into the CFK equation and solved for $\mathrm{DL}_{\mathrm{CO}}$ using a computer program generated in MATLAB. We then input the estimated $\mathrm{DL}_{\mathrm{CO}}$ and the $\mathrm{COHb}$ level measured after 20 minutes into the $\mathrm{CFK}$ equation and calculated the $\mathrm{COHb}$ level predicted after $\mathrm{CO}$ exposure for time $=60,75$, and 90 minutes.

Additional secondary endpoints included $\mathrm{PaO}_{2} / \mathrm{FiO}_{2}$, OI, and lactate on days 1-5; LIS and SOFA score on days 1-5 and day 7; vasopressor-free days, VFDs, and ICU-free days to day 28; hospital mortality; and biomarker levels.

Statistics. Baseline demographic and clinical characteristics were compared by Mann-Whitney $U$, Kruskal-Wallis 1-way ANOVA, or Fisher's exact tests. COHb levels were analyzed among treatment groups by 2 -way ANOVA followed by Tukey's post hoc test. Repeated-measures ANOVA was used to analyze (i) $\Delta$ 90 minute $\mathrm{COHb}$ over 5 days, (ii) group difference between placebo and $\mathrm{CO}$ treatment, and (iii) interaction between day and group effect. The accuracy of the CFK equation in predicting $\mathrm{COHb}$ levels was analyzed by Spearman's correlation and Bland-Altman plots using measured versus predicted $\mathrm{COHb}$ levels and modeled using linear regression. $\mathrm{LIS}, \mathrm{PaO}_{2} / \mathrm{FiO}_{2}$, lactate, and OI were compared among the treatment groups and over time using a mixed model to account for fixed and random effects, while SOFA scores were compared via ordinal logistic analysis (JMP version 12.0, SAS Institute).

Due to the small sample size, our study was powered to detect only common safety and toxicity outcomes, with a low probability of detecting rare safety events. We did not establish formal criteria for evaluation of excess AE frequency; however, our internal SRC and external DSMB were empowered to terminate the study based on the nature and seriousness of any AE.

All biomarkers were analyzed on a $\log _{10}$ scale to correct for right skewness and then transformed back for interpretability. Given the variation across patients in baseline biomarker levels, within-patient changes were calculated by determining the difference between pretreatment levels on day 1 and posttreatment 
(90 minutes) levels on subsequent days. Ten patients received treatment on both days 1 and 2 and were included in the primary biomarker analysis. Subjects 9 and 10 did not receive treatment on study day 1 and therefore were excluded from the primary biomarker analysis. Mean changes in biomarkers among subjects receiving $100 \mathrm{ppm}$ and $200 \mathrm{ppm}$ iCO were compared with changes in subjects receiving placebo air in a pairwise manner using 2-sided $t$ tests, with nonparametric tests performed for sensitivity analyses. Data were analyzed using Prism 7.0 (GraphPad), R (R Foundation for Statistical Computing), or SAS 9.4. All $P$ values were 2 tailed, and statistical significance was accepted at $P<0.05$. The remaining analyses are descriptive. This study is registered with ClinicalTrials.gov (NCT02425579).

Study approval. Study approval was obtained from the Partners Human Research Committee, Weill Cornell Medical College IRB, and Duke University Health System IRB for Clinical Investigations. Prior to inclusion in the study, written informed consent was obtained from eligible participants by a physician investigator after discussion with the patient or LAR or surrogate.

\section{Author contributions}

LEF, MAP, DRH, EP, KEWW, BDK, RSH, JDD, JAE, PBD, DAB, CAP, RMB, BTT, and AMKC were study investigators. RMB and BTT led the Data Coordinating Center. LEF, MAP, EP, KEWW, BDK, RSH, RM, SH, CNS, CAP, RMB, BTT, and AMKC participated in the study design. RM and SH prepared the randomization schedule. $\mathrm{LEF}, \mathrm{DRH}, \mathrm{RSH}$, and TW participated in the design and programming of the CFK equation program. LEF, MAP, DBB, DRH, KEWW, BDK, RSH, JDD, AH, JAE, PBD, DAB, SL, MB, AIS, PFN, MTK, MJM, MAPP, and EF enrolled and treated patients and/or collected data. DBB, $\mathrm{EP}, \mathrm{AH}$, and MTK were responsible for monitoring. KTS and $\mathrm{KN}$ performed the biomarker assays and analysis. LEF, MAP, KEWW, BDK, RSH, RM, KN, CO, AH, SH, CAP, RMB, BTT, and AMKC analyzed and/or interpreted the data. LEF drafted the manuscript. All authors read, revised, and approved the manuscript. LEF and AMKC had full access to all of the data in the study and take full responsibility for the integrity of the data and accuracy of the data analysis.

\section{Acknowledgments}

This work was supported by funding from the NIH, including P01HL108801, KL2TR002385, K08HL130557, and K08GM102695. We would like to thank the Data and Safety Monitoring Board (Kathleen Liu [chair], UCSF; Mark Gladwin, University of Pittsburgh Medical Center; and Marc Moss, University of Colorado Denver). We would like to thank Chad Steele for assistance with Luminex assays. We also wish to thank our patients for their generous participation in this study.

Address correspondence to: Laura E. Fredenburgh, Division of Pulmonary and Critical Care Medicine, Brigham and Women's Hospital, 75 Francis Street, Boston, Massachusetts 02115, USA. Phone: 617.525.9563; Email: 1fredenburgh@rics.bwh.harvard.edu. Or to: Augustine M.K. Choi, Weill Cornell Medicine, 1300 York Avenue, Suite F-113 Box 83, New York, New York 10065, USA. Phone: 212.746.6005; Email: amc2056@med.cornell.edu.

JAE's present address is: Division of Pulmonary, Critical Care and Sleep Medicine, The Ohio State University Wexner Medical Center, Columbus, Ohio, USA.

DBB's present address is: Department of Anesthesia, Critical Care and Pain Medicine, Massachusetts General Hospital, Boston, Massachusetts, USA.

1. Bellani G, et al. Epidemiology, patterns of care, and mortality for patients with acute respiratory distress syndrome in intensive care units in 50 countries. JAMA. 2016;315(8):788-800.

2. Angus DC, Linde-Zwirble WT, Lidicker J, Clermont G, Carcillo J, Pinsky MR. Epidemiology of severe sepsis in the United States: analysis of incidence, outcome, and associated costs of care. Crit Care Med. 2001;29(7):1303-1310.

3. Epstein L, Dantes R, Magill S, Fiore A. Varying estimates of sepsis mortality using death certificates and administrative codes — United States, 1999-2014. MMWR Morb Mortal Wkly Rep. 2016;65(13):342-345.

4. Acute Respiratory Distress Syndrome Network, et al. Ventilation with lower tidal volumes as compared with traditional tidal volumes for acute lung injury and the acute respiratory distress syndrome. N Engl J Med. 2000;342(18):1301-1308.

5. Guérin C, et al. Prone positioning in severe acute respiratory distress syndrome. N Engl J Med. 2013;368(23):2159-2168.

6. Papazian L, et al. Neuromuscular blockers in early acute respiratory distress syndrome. N Engl J Med. 2010;363(12):1107-1116. 
7. Dolinay T, Szilasi M, Liu M, Choi AM. Inhaled carbon monoxide confers antiinflammatory effects against ventilator-induced lung injury. Am J Respir Crit Care Med. 2004;170(6):613-620.

8. Otterbein LE, Mantell LL, Choi AM. Carbon monoxide provides protection against hyperoxic lung injury. Am J Physiol. 1999;276(4 Pt 1):L688-L694.

9. Fredenburgh LE, et al. Effects of inhaled CO administration on acute lung injury in baboons with pneumococcal pneumonia. Am J Physiol Lung Cell Mol Physiol. 2015;309(8):L834-L846.

10. Otterbein LE, et al. Carbon monoxide has anti-inflammatory effects involving the mitogen-activated protein kinase pathway. Nat Med. 2000;6(4):422-428.

11. Lee S, et al. Carbon monoxide confers protection in sepsis by enhancing beclin 1-dependent autophagy and phagocytosis. Antioxid Redox Signal. 2014;20(3):432-442.

12. Mitchell LA, Channell MM, Royer CM, Ryter SW, Choi AM, McDonald JD. Evaluation of inhaled carbon monoxide as an anti-inflammatory therapy in a nonhuman primate model of lung inflammation. Am J Physiol Lung Cell Mol Physiol. 2010;299(6):L891-L897.

13. Ryter SW, Ma KC, Choi AMK. Carbon monoxide in lung cell physiology and disease. Am J Physiol, Cell Physiol. 2018;314(2):C211-C227.

14. Nakahira K, Choi AM. Carbon monoxide in the treatment of sepsis. Am J Physiol Lung Cell Mol Physiol. 2015;309(12):L1387-L1393.

15. Lee SJ, et al. Carbon monoxide activates autophagy via mitochondrial reactive oxygen species formation. Am J Respir Cell Mol Biol. 2011;45(4):867-873.

16. Jung SS, et al. Carbon monoxide negatively regulates NLRP3 inflammasome activation in macrophages. Am J Physiol Lung Cell Mol Physiol. 2015;308(10):L1058-L1067.

17. Pecorella SR, et al. The HO-1/CO system regulates mitochondrial-capillary density relationships in human skeletal muscle. Am J Physiol Lung Cell Mol Physiol. 2015;309(8):L857-L871.

18. Chiang $\mathrm{N}$, et al. Inhaled carbon monoxide accelerates resolution of inflammation via unique proresolving mediator-heme oxygenase-1 circuits. J Immunol. 2013;190(12):6378-6388.

19. Dalli J, et al. The Regulation of proresolving lipid mediator profiles in baboon pneumonia by inhaled carbon monoxide. $A m J$ Respir Cell Mol Biol. 2015;53(3):314-325.

20. Hausberg M, Somers VK. Neural circulatory responses to carbon monoxide in healthy humans. Hypertension. 1997;29(5):1114-1118

21. Mayr FB, et al. Effects of carbon monoxide inhalation during experimental endotoxemia in humans. Am J Respir Crit Care Med. 2005;171(4):354-360.

22. Peterson JE, Stewart RD. Predicting the carboxyhemoglobin levels resulting from carbon monoxide exposures. J Appl Physiol. 1975;39(4):633-638.

23. Stewart RD, Peterson JE, Baretta ED, Bachand RT, Hosko MJ, Herrmann AA. Experimental human exposure to carbon monoxide. Arch Environ Health. 1970;21(2):154-164

24. Zevin S, Saunders S, Gourlay SG, Jacob P, Benowitz NL. Cardiovascular effects of carbon monoxide and cigarette smoking. $J$ Am Coll Cardiol. 2001;38(6):1633-1638.

25. Rhodes MA, et al. Carbon monoxide, skeletal muscle oxidative stress, and mitochondrial biogenesis in humans. Am JPhysiol Heart Circ Physiol. 2009;297(1):H392-H399.

26. Ren X, Dorrington KL, Robbins PA. Respiratory control in humans after $8 \mathrm{~h}$ of lowered arterial PO2, hemodilution, or carboxyhemoglobinemia. J Appl Physiol. 2001;90(4):1189-1195.

27. Bathoorn E, et al. Anti-inflammatory effects of inhaled carbon monoxide in patients with COPD: a pilot study. Eur Respir J. 2007;30(6):1131-1137

28. Rosas IO, et al. A phase ii clinical trial of low-dose inhaled carbon monoxide in idiopathic pulmonary fibrosis. Chest. 2018;153(1):94-104

29. Hauck H, Neuberger M. Carbon monoxide uptake and the resulting carboxyhemoglobin in man. Eur J Appl Physiol Occup Physiol. $1984 ; 53(2): 186-190$.

30. Tikuisis P, Kane DM, McLellan TM, Buick F, Fairburn SM. Rate of formation of carboxyhemoglobin in exercising humans exposed to carbon monoxide. J Appl Physiol. 1992;72(4):1311-1319.

31. Shinohara M, et al. Cell-cell interactions and bronchoconstrictor eicosanoid reduction with inhaled carbon monoxide and resolvin D1. Am J Physiol Lung Cell Mol Physiol. 2014;307(10):L746-L757.

32. Mumby S, et al. Lung heme oxygenase-1 is elevated in acute respiratory distress syndrome. Crit Care Med. 2004;32(5):1130-1135

33. Weaver LK, Howe S, Hopkins R, Chan KJ. Carboxyhemoglobin half-life in carbon monoxide-poisoned patients treated with 100\% oxygen at atmospheric pressure. Chest. 2000;117(3):801-808.

34. Ferreira FL, Bota DP, Bross A, Mélot C, Vincent JL. Serial evaluation of the SOFA score to predict outcome in critically ill patients. JAMA. 2001;286(14):1754-1758.

35. Kamel H, et al. Diagnostic yield of electroencephalography in the medical and surgical intensive care unit. Neurocrit Care. $2013 ; 19(3): 336-341$

36. Craig DG, et al. The sequential organ failure assessment (SOFA) score is an effective triage marker following staggered paracetamol (acetaminophen) overdose. Aliment Pharmacol Ther. 2012;35(12):1408-1415

37. Dolinay T, et al. Inflammasome-regulated cytokines are critical mediators of acute lung injury. Am J Respir Crit Care Med. 2012;185(11):1225-1234.

38. Nakahira K, et al. Circulating mitochondrial DNA in patients in the ICU as a marker of mortality: derivation and validation. PLoS Med. 2013;10(12):e1001577.

39. Ma KC, et al. Circulating RIPK3 levels are associated with mortality and organ failure during critical illness. JCI Insight. 2018;3(13):e99692.

40. Brealey D, et al. Association between mitochondrial dysfunction and severity and outcome of septic shock. Lancet. 2002;360(9328):219-223.

41. Nakahira K, et al. Autophagy proteins regulate innate immune responses by inhibiting the release of mitochondrial DNA mediated by the NALP3 inflammasome. Nat Immunol. 2011;12(3):222-230. 
42. Harrington JS, Choi AMK, Nakahira K. Mitochondrial DNA in Sepsis. Curr Opin Crit Care. 2017;23(4):284-290.

43. Nakahira K, Hisata S, Choi AM. The roles of mitochondrial damage-associated molecular patterns in diseases. Antioxid Redox Signal. 2015;23(17):1329-1350.

44. Carré JE, et al. Survival in critical illness is associated with early activation of mitochondrial biogenesis. Am J Respir Crit Care Med. 2010;182(6):745-751.

45. Ma KC, Schenck EJ, Pabon MA, Choi AMK. The role of danger signals in the pathogenesis and perpetuation of critical illness. Am J Respir Crit Care Med. 2018;197(3):300-309.

46. Zuckerbraun BS, et al. Carbon monoxide reverses established pulmonary hypertension. J Exp Med. 2006;203(9):2109-2119.

47. Otterbein LE, et al. Carbon monoxide suppresses arteriosclerotic lesions associated with chronic graft rejection and with balloon injury. Nat Med. 2003;9(2):183-190.

48. Habets MGJL, van Delden JJM, Bredenoord AL. The unique status of first-in-human studies: strengthening the social value requirement. Drug Discov Today. 2017;22(2):471-475.

49. [No authors listed]. Guidance for industry: estimating the maximum safe starting dose in initial clinical trials for therapeutics in adult healthy volunteers. Food and Drug Administration. https://www.fda.gov/downloads/drugs/guidancecomplianceregulatoryinformation/guidances/ucm078932.pdf. Accessed November 7, 2018.

50. Piantadosi CA, et al. Heme oxygenase-1 couples activation of mitochondrial biogenesis to anti-inflammatory cytokine expression. J Biol Chem. 2011;286(18):16374-16385.

51. MacGarvey NC, et al. Activation of mitochondrial biogenesis by heme oxygenase-1-mediated NF-E2-related factor-2 induction rescues mice from lethal Staphylococcus aureus sepsis. Am J Respir Crit Care Med. 2012;185(8):851-861.

52. Ghanta S, et al. Mesenchymal stromal cells deficient in autophagy proteins are susceptible to oxidative injury and mitochondrial dysfunction. Am J Respir Cell Mol Biol. 2017;56(3):300-309.

53. ARDS Definition Task Force, et al. Acute respiratory distress syndrome: the Berlin Definition. JAMA. 2012;307(23):2526-2533.

54. Singer M, et al. The Third International Consensus Definitions for Sepsis and Septic Shock (Sepsis-3). JAMA. 2016;315(8):801-810.

55. Levy MM, et al. 2001 SCCM/ESICM/ACCP/ATS/SIS International Sepsis Definitions Conference. Crit Care Med. 2003;31(4):1250-1256.

56. [No authors listed]. American College of Chest Physicians/Society of Critical Care Medicine Consensus Conference: definitions for sepsis and organ failure and guidelines for the use of innovative therapies in sepsis. Crit Care Med. 1992;20(6):864-874.

57. Hébert PC, et al. A multicenter, randomized, controlled clinical trial of transfusion requirements in critical care. Transfusion requirements in critical care investigators, Canadian Critical Care Trials Group. N Engl J Med. 1999;340(6):409-417.

58. Pocock S. Clinical Trials: A Practical Approach. New York, New York, USA: Wiley; 1984.

59. National Heart, Lung, Blood Institute Acute Respiratory Distress Syndrome (ARDS) Clinical Trials Network, et al. Comparison of two fluid-management strategies in acute lung injury. $N$ Engl J Med. 2006;354(24):2564-2575.

60. Meduri GU, et al. Methylprednisolone infusion in early severe ARDS: results of a randomized controlled trial. Chest. 2007;131(4):954-963.

61. Meduri GU, et al. Effect of prolonged methylprednisolone therapy in unresolving acute respiratory distress syndrome: a randomized controlled trial. JAMA. 1998;280(2):159-165.

62. Murray JF, Matthay MA, Luce JM, Flick MR. An expanded definition of the adult respiratory distress syndrome. Am Rev Respir Dis. 1988;138(3):720-723.

63. Wilson JG, et al. Mesenchymal stem (stromal) cells for treatment of ARDS: a phase 1 clinical trial. Lancet Respir Med. 2015;3(1):24-32.

64. Brown SM, et al. Nonlinear imputation of $\mathrm{PaO} 2 / \mathrm{FIO} 2$ from $\mathrm{SpO} 2 / \mathrm{FIO} 2$ among mechanically ventilated patients in the ICU: a prospective, observational study. Crit Care Med. 2017;45(8):1317-1324.

65. Brown SM, et al. Nonlinear imputation of Pao2/Fio2 from Spo2/Fio2 among patients with acute respiratory distress syndrome. Chest. 2016;150(2):307-313.

66. Brown SM, et al. Survival after shock requiring high-dose vasopressor therapy. Chest. 2013;143(3):664-671.

67. Knox DB, Lanspa MJ, Kuttler KG, Brewer SC, Brown SM. Phenotypic clusters within sepsis-associated multiple organ dysfunction syndrome. Intensive Care Med. 2015;41(5):814-822.

68. Qing DY, et al. Red blood cells induce necroptosis of lung endothelial cells and increase susceptibility to lung inflammation. Am J Respir Crit Care Med. 2014;190(11):1243-1254.

69. Coburn RF, Forster RE, Kane PB. Considerations of the physiological variables that determine the blood carboxyhemoglobin concentration in man. J Clin Invest. 1965;44(11):1899-1910. 